\title{
The Radio Properties of Optically Selected Quasars. III. Comparison between Optical and X-Ray Selected Samples 1
}

\author{
Eric J. Hooper, Chris D. Impey \\ Steward Observatory, University of Arizona, Tucson, AZ 85721 \\ ehooper@as.arizona.edu, cimpey@as.arizona.edu \\ Craig B. Foltz \\ Multiple Mirror Telescope Observatory, University of Arizona, Tucson, AZ 85721 \\ cfoltz@as.arizona.edu \\ and \\ Paul C. Hewett \\ Institute of Astronomy, Madingley Road, CB3 0HA Cambridge, United Kingdom \\ phewett@mail.ast.cam.ac.uk
}

\begin{abstract}
A sample of 103 quasars from the Large Bright Quasar Survey (LBQS) has been observed with the VLA at $8.4 \mathrm{GHz}$ to study the evolution of the radio luminosity distribution and its dependence on absolute magnitude. Radio data from pointed observations are now available for 359 of the 1055 LBQS quasars. The radio-loud fraction is constant at $\approx 10 \%$ over the absolute magnitude range $-28 \lesssim M_{B} \lesssim-23$, and it rises to $\sim 20 \%\left(\log R_{8.4}>1\right)$ or $\sim 35 \%\left(\log L_{8.4}>25\right)$ at the brightest absolute magnitudes in the sample. This nearly flat distribution differs markedly from those of the optically selected Palomar-Green (PG) Bright Quasar Survey and the X-ray selected Extended Medium Sensitivity Survey (EMSS), both of which have lower radio-loud fractions for absolute magnitudes fainter than $M_{B}=-24$ and higher fractions at brighter magnitudes. The reason for the high radio-loud fraction at bright absolute magnitudes in the PG, compared to the LBQS and other optically selected quasar surveys, is unknown. The trend of increasing radio-loud fraction with absolute magnitude in the EMSS is due at least in part to a correlation between X-ray and radio luminosity. Combining the LBQS data with radio studies of high-redshift quasars leads to the conclusion that the radio-loud fraction in optically selected quasars does not appear to evolve significantly, aside from a modest increase at $z \sim 1$, from $z=0.2$ to redshifts approaching 5 , a result that is contrary to previous studies which found a decrease in radio-loud fraction with increasing redshift by comparing the low- $z$ fraction in the PG to higher redshift samples.
\end{abstract}

\footnotetext{
${ }^{1}$ Some of the observations reported here were obtained with the Multiple Mirror Telescope, a facility operated jointly by the University of Arizona and the Smithsonian Institution.
} 
Subject headings: quasars: general - radio continuum: galaxies - surveys

\section{Introduction}

Optical and near-UV light from quasars is comprised primarily of thermal components and emission lines, with some contribution from synchrotron radiation. The source of the optical continuum and broad lines in the standard black hole model for quasars (e.g., Rees 1984) is an accretion disk and centrally concentrated hot gas clouds, respectively. In this model, optical luminosity is closely tied to the accretion rate and the mass of the central engine.

Radiation at radio frequencies is predominantly due to synchrotron processes, which depend on the energy distribution of relativistic particles and magnetic field strength. The ultimate power source for particle acceleration and magnetic field production is presumably the same gravitational engine producing the optical flux, but the relationship is not straightforward. Radio and optical data for an ensemble of quasars should place constraints on the connections between the fueling of the black hole, the gas and dust in the vicinity of the central engine, and the particle acceleration mechanism.

The relationship between quasar radio and optical emission was studied initially using radio-selected objects, which generally had high radio luminosities due to the relatively low sensitivity limits of the surveys. Sandage (1965) determined that not all quasars are powerful radio sources and hence detectable in radio surveys. Since then, in addition to radio surveys, radio follow-up observations have been made of surveys conducted in the optical (e.g., Sramek \& Weedman 1980; Condon et al. 1981; Marshall 1987; Miller, Peacock, \& Mead 1990; Schmidt et al. 1995) and other wave bands. Targeted radio observations of quasars selected by other means typically go deeper than the radio surveys. As a result the median radio luminosity of these samples is lower, but they usually contain few of the very powerful objects common in radio surveys. Taken together the two survey methods have detected quasars with a range of more than 6 orders of magnitude in radio luminosity. This wide span of radio power has commonly been divided into two regimes, radio-loud and radio-quiet.

Two definitions of radio-loud are generally employed, one based on radio luminosity, and the other on the ratio, $R_{\nu}$, of radio luminosity at frequency $\nu$ to optical or near-ultraviolet luminosity. These different definitions produce the same classification for most quasars, except at low and high optical luminosities. The empirical basis for this was discussed extensively in Stocke et al. (1992) and in Hooper et al. (1995; hereafter Paper II). Peacock, Miller, \& Longair (1986) explored the implications of using radio luminosity or a radio-to-optical luminosity ratio as the parameter of radio strength in a joint radio-optical luminosity function. The analysis in the present work is conducted using both definitions. 
Many studies have concluded that the fraction of radio-loud quasars decreases with increasing redshifts by comparing the radio observations of the predominantly low-redshift Palomar-Green optical quasar survey (Kellermann et al. 1989, hereafter PG), which has a high radio-loud fraction, to higher redshift optically selected samples with relatively fewer radio-loud quasars (Peacock et al. 1986; Miller et al. 1990; Schneider et al. 1992; Visnovsky et al. 1992). However, there is no strong evidence for evolution for $z>1$ if the PG is excluded from the ensemble of optically selected samples (La Franca et al. 1994). A sample of quasars from the Large Bright Quasar Survey (LBQS; Hewett, Foltz, \& Chaffee 1995) in the redshift range $0.2<z<3.4$ did not show the strong evolution in radio-loud fraction inferred from comparisons of the $\mathrm{PG}$ with other surveys (Paper II). These results indicate that the PG has an anomalously high radio-loud fraction (at bright absolute magnitudes) compared to other optically selected quasar samples. This difference remains unexplained.

Peacock et al. (1986) noted that strong radio emitters were found to be relatively rare at absolute blue magnitudes $\left(M_{B}\right)$ fainter than -24 . These authors proposed a simple and plausible selection effect, arising from the classification of active galactic nuclei (AGN) as either quasars or radio galaxies, to explain this observation. If radio-loud AGN reside in large bright elliptical galaxies, and radio-quiet AGN have less luminous spiral host galaxies (see, e.g., Smith et al. 1986; Véron-Cetty \& Woltjer 1990; Hutchings et al.; however, there are indications that some radio-quiet quasars reside in elliptical host galaxies: e.g., Disney et al. 1995; Bahcall, Kirhakos, \& Schneider 1996), powerful radio sources with optically weak nuclei would be classified as radio galaxies in radio surveys. They would not be selected by optical surveys because of the low contrast between the quasar light and the relatively bright, red, and extended stellar component. In fact, just such a decrease in the fraction of radio-loud quasars with $M_{B}>-24$ has been seen directly in follow-up radio data to optical and X-ray surveys. The effect is highly significant in the PG survey (see also Padovani 1993; La Franca et al. 1994), and a sharp decrease in radio-loud fraction at the same optical luminosity is present among the quasars selected at X-ray wavelengths by the Extended Medium Sensitivity Survey (EMSS; Della Ceca et al. 1994). In apparent support of this conclusion, no radio-loud quasars fainter than $M_{B}=-24$ were found in a sample from the LBQS discussed in Paper II. The probability of selecting a quasar using the LBQS criteria is quantifiable in terms of quasar spectral energy distribution, absolute magnitude, redshift, and brightness relative to the host galaxy, an advantage over many existing surveys. This probability distribution was used to test the selection effect hypothesis of Peacock et al. (1986), which was found to not explain the trend seen in the sample drawn from the LBQS.

Further analysis of the radio properties of optically selected quasars with absolute magnitudes near $M_{B}=-24$ was the primary motivation for obtaining the data presented in the current work, which is the third paper in a series examining the radio properties of the LBQS. Paper I in the series (Visnovsky et al. 1992) presented radio data for 124 high redshift $(1.0<z<3.0)$ LBQS quasars, selected as a comparison sample for the predominantly low redshift PG survey. The parameter $R_{8.4}$ was found to be independent of both redshift and absolute magnitude in this 
subsample, and the distribution of $\log R_{8.4}$ appeared to be bimodal. The radio-loud fraction was lower than in the PG sample, from which it was inferred that the fraction evolves. One of the principal motivations at that point for expanding the LBQS radio sample was to cover the full redshift range of the LBQS $(0.2<z<3.4)$, in order to independently investigate the evolution of the radio-loud fraction using a single well-defined survey. An additional 132 LBQS quasars were added to the radio sample in Paper II to accomplish this. The optically brightest quasars at each redshift were selected for Papers I and II in order to study the radio properties at the lowest values of $R_{8.4}$ possible for a given radio flux limit. A more detailed discussion of sample selection is given in Section 2.1. The radio-loud fraction in the Paper II sample was nearly constant at $\sim 10 \%$ from redshift 0.2 to 3.4 , and, with the addition of high- $z$ samples from the literature, showed no evolution to a redshift of almost 5 . The fraction was also independent of absolute blue magnitude in the range $-27.5<M_{B}<-24$. A higher radio-loud fraction was found at the brightest absolute magnitudes. As discussed above, there were no radio-loud quasars with $M_{B}>-24$.

The change in radio-loud fraction of LBQS quasars at faint absolute magnitudes reported in Paper II, while significant at the $97 \%$ confidence level, was based on only 20 quasars with $M_{B}$ fainter than -24 . Absolute magnitude errors were 0.3 to 0.4 for $M_{B} \sim-24$. Given the survey's relative insensitivity to selection bias against optically weak, radio-loud quasars (see Paper II and Hewett et al. 1995 for details), it is important to expand the radio database of LBQS quasars and to reduce the magnitude uncertainties of radio-loud objects with absolute magnitudes near

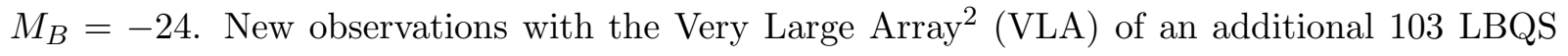
quasars with absolute magnitudes in the range $-25<M_{B}<-23$ and redshifts $0.2<z<1.1$ are presented in this paper, along with improved magnitude determinations for objects at faint optical $M_{B}$ (Section 2). The latest data are combined with those from Papers I and II to form one of the largest samples of sensitive radio observations of quasars selected at other wavelengths in a single survey. The distribution of radio luminosity as a function of $M_{B}$ is analyzed and compared to the PG and EMSS samples (Section 3), and the evolution of the radio properties of these three samples is explored in Section 4. Models proposed to explain the radio luminosity distribution as a function of absolute magnitude are discussed in Section 5. Section 6 contains a summary of the major results.

\footnotetext{
${ }^{2}$ The Very Large Array (VLA) of the National Radio Astronomy Observatory is operated by Associated Universities, Inc., under a cooperative agreement with the National Science Foundation.
} 


\section{Observations and Data Reduction}

Radio observations of an additional sample of LBQS quasars and improved optical data for a subset of the radio-loud quasars are presented and discussed below. Rest-frame $8.4 \mathrm{GHz}$ luminosities $\left(L_{8.4}\right)$ and absolute blue magnitudes $\left(M_{B}\right)$ were calculated using $H_{0}=50 \mathrm{~km} \mathrm{~s}^{-1}$ $\mathrm{Mpc}^{-1}$ and $q_{0}=0.5$. The luminosity ratio, $R_{8.4}$, is $L_{8.4}$ divided by the average luminosity over the $B$ passband.

The radio luminosities were derived from observed fluxes assuming a radio spectral index $\alpha=-0.5\left(f_{\nu} \propto \nu^{\alpha}\right)$. Optical $k$-corrections for the majority of the quasars in the sample were calculated from the composite quasar spectrum of Francis et al. (1991). Details of these calculations and attendant error analysis are given in Paper II. The absolute magnitude errors, which are conservative overestimates, are typically \pm 0.4 mag for $M_{B} \approx-24$, rising at brighter $M_{B}$ (higher redshifts) to \pm 0.9 . These errors are the quadrature sum of three components: 1 ) uncertainty in the $k$-correction, the dominant source of error for most of the quasars in the sample, particularly at high redshifts; 2) optical variability of the quasars over the time interval between the optical and radio observations; and 3) a photometric imprecision of $\approx 0.15$ mag in the original $B_{J}$ magnitudes determined from Schmidt photographic plates (Hewett et al. 1995), which is the smallest contributor to the total uncertainty, although still significant at faint absolute magnitudes. While the resulting precision is adequate for determining large-scale trends in a substantial data set, a detailed view of the change in radio-loud fraction around $M_{B}=-24$ is difficult with the current magnitude uncertainties, given the small number of radio-loud quasars. Each of these uncertainties was reduced for several radio-loud quasars with absolute magnitudes near $M_{B}=-24$ by obtaining improved optical spectra and multicolor CCD photometry at an epoch closer to that of the radio data.

\subsection{Sample Selection}

Each subsample of the LBQS selected for observing with the VLA was chosen to address specific scientific questions, as discussed in the introduction. Neither the individual subsamples nor the combined set of radio data are representative of the LBQS as a whole, in the sense that the distributions of $B, z$, and $M_{B}$ are different for the radio sample and the remaining LBQS quasars (see Figure 1 in Paper II). The current sample is simply that from Paper II with the addition of many of the remaining LBQS quasars in the magnitude range $-25<M_{B}<-23$. The radio sample now contains 146/276 (53\%) of all LBQS quasars in this absolute magnitude range. The 103 new observations are nearly equally divided about $M_{B}=-24$. Figure 1 demonstrates the effects of the various selection criteria on the radio sample. Absolute blue magnitude is plotted against redshift, with individual radio detections and upper limits indicated by filled circles and horizontal lines, respectively, and the Y-shaped symbols represent LBQS quasars without radio data. All of the quasars without radio data are plotted for $z>2.5$, but, due to the large numbers 
of such objects at lower redshifts, only $1 / 3$ of them with $z<2.5$ are plotted.

Figure 1 shows that, for $M_{B}<-25$, the radio sample lies in the brighter portion of the region of absolute magnitude/redshift space occupied by the LBQS. This effect is due to the selection priorities of Papers I and II, which resulted in the exclusion of almost all LBQS quasars with $B>18.4$ from those samples. There is a thin strip corresponding to $B>18.4$ in which there are no radio data, and the radio properties of the LBQS are formally unconstrained for these combinations of $z$ and $M_{B}$. However, it is unlikely that the radio properties are radically different in this unsampled region. The strip is narrow, typically half a magnitude at any redshift. The difference between the brightest members of the radio sample and the remaining LBQS quasars was measured in a series of adjoining redshift intervals of width 0.2 over the range $1.1<z<3.3$. The average magnitude difference is $0.55 \mathrm{mag}$, with an rms dispersion about the mean of 0.15 mag. The unsampled region spans between $125 \mathrm{Myr}$ and $1 \mathrm{Gyr}$ of lookback time. Therefore, any major differences between the sample and the unsampled region would have to occur over a luminosity interval of $0.5 \mathrm{mag}$ and $\leq 1 \mathrm{Gyr}$ in lookback time. These are small intervals for major changes, given that the radio properties of LBQS quasars for which data exist are nearly constant

over 5 magnitudes and 8 Gyr of lookback time (see Sections 3 and 国). Note that, for every value of redshift and absolute magnitude in the unsampled strip, there are radio data at a different $z / M_{B}$.

The unsampled region extended over all absolute magnitudes in Paper II, but it ends at $M_{B}=-25$ in the present work due to the addition of the new radio data in the range $-25<M_{B}<-23$. The radio sample now covers the same region of absolute magnitude/redshift space as the entire LBQS in this absolute magnitude range. No radio data are available for LBQS quasars fainter than $M_{B}=-22.7$ (Figure 1), but this does not affect the conclusions regarding the radio properties of the brighter quasars in the sample.

\subsection{Radio}

All of the radio observations of LBQS quasars were obtained with the VLA at a frequency of $8.4 \mathrm{GHz}$. The latest group of 103 quasars, presented here, were observed on UT 1993 June 4 and 25 in the $\mathrm{B} / \mathrm{C}$ and $\mathrm{C}$ array configurations, respectively. Exposure times were about 2 minutes for all objects. Standard AIPS software was employed to produce dirty total-intensity (Stokes I) maps for all fields. Those containing a strong source were subsequently CLEANed.

A detailed description of the noise analysis and source detection procedure used for these maps was presented in Paper II, but the principal steps are summarized here. Radio flux at any point on a map was determined by fitting the core of the corresponding beam to the region around the desired map location. Noise levels were determined by performing the fitting procedure on a random sample of points across each map. Subsequently, a circle of radius $2^{\prime \prime}$ at the center of the map was searched for radio flux exceeding a detection threshold of three times the rms noise value.

A small number of spurious detections are expected near the $3 \sigma$ detection limit. The 
probability of obtaining a $3 \sigma$ or greater positive fluctuation at a given position on an apparently empty map in the latest set of observations is $1.9 \times 10^{-3}, 1.4$ times greater than predicted by a Gaussian noise model using the measured rms. The excess of positive fluctuations may be due to weak sources in the maps, or it may be due to part of the sidelobe patterns from unknown off-map sources. The chance of a spurious detection in a map is this probability multiplied by the number of independent sampling points within the $2^{\prime \prime}$ search radius. The average number of independent sample points per search disk for the current dataset is 3.4, giving an expected total of $\left(1.9 \times 10^{-3}\right)(3.4) 103 \approx 0.7$ spurious detections among the 103 maps. The expected number of spurious detections in the previous radio data sets is 2.4 (Paper II), for a total of 3.1 among 61 recorded detections from 359 observed fields.

The search radius was chosen to match the astrometric accuracy of the LBQS, estimated to

be $\lesssim 2^{\prime \prime}$ for the original quasar positions. The results were checked by reanalyzing the data with a 4..5 search radius to look for larger positional offsets. Every strong source (flux $>7 \sigma$, where $\sigma$ is the rms noise) found using the larger search radius is within $2^{\prime \prime}$ of the map center, and the greatest distance from the center of any detected source is 2 ." 4 (1322-0204, a 3.8 $\sigma$ detection). One quasar not detected using a $2^{\prime \prime}$ radius had a $>3 \sigma$ positive fluctuation within a 4 ".5 radius. The expected number of additional spurious detections using the larger radius in 103 maps is $\sim 2.7$. These tests indicate that a $2^{\prime \prime}$ search radius is adequate, and this value was used to determine the LBQS radio fluxes.

Radio and optical data for each quasar in the current data set, as well as quasars listed in Paper II with new optical data, are listed in Table 1 in the following format:

Column (1): Object name, listed in order of increasing right ascension.

Column (2): Apparent B-magnitude derived from observed $B_{J}$ (Hewett et al. 1995) as described in Paper II, or, for 5 quasars flagged in the table, measured directly (Section 2.3).

Column (3): Redshift from Hewett et al. (1995).

Column (4): Absolute B-magnitude.

Column (5): Flux density (mJy) at $8.4 \mathrm{GHz}$ and $1 \sigma$ error, or $3 \sigma$ upper limit.

Column (6): Logarithm of the ratio of $8.4 \mathrm{GHz}$ luminosity $\left(\mathrm{W} \mathrm{Hz}^{-1}\right)$ to optical luminosity ( $\mathrm{W} \mathrm{Hz}^{-1}$ ) averaged over the $B$ passband; $R_{8.4}=L_{8.4} / L_{B}$.

Column (7): Logarithm of $8.4 \mathrm{GHz}$ luminosity ( $\left.\mathrm{W} \mathrm{Hz}^{-1}\right), \log L_{8.4}$.

\subsection{Optical}

Many existing spectra of LBQS quasars suffer from systematic light loss in the blue due to several instrumental effects and from poor signal-to-noise ratio. Higher quality long slit CCD 
spectra were obtained with the Multiple Mirror Telescope (MMT) for 10 radio-loud LBQS quasars on the night of UT $1995 \mathrm{Feb}$ 5. The new data, which cover a wavelength range $3400-6900 \AA$, were reduced using standard procedures. The normalization of the flux scale is unreliable, due to a narrow slit and thin cirrus during the observations, but the relative flux calibration is unaffected, since the long axis of the slit was maintained parallel to the direction of atmospheric dispersion.

Improved spectra were not obtained for three radio-loud quasars with $M_{B}$ near -24 (1430-0046, 2348+0210, and 2351-0036), so archival spectra were analyzed. The spectrum for the latter object does not appear to suffer from the instrumental blue light loss mentioned previously. The other two archival spectra show downturns blueward of $4000 \AA$. This wavelength region was excluded from spectral index determinations.

A spectral slope was determined for each of the 13 quasars using the ratios of fluxes measured in pairs of fixed rest frame continuum windows chosen to avoid prominent emission lines (Francis 1993). Only one pair of windows was observable in each spectrum, either 2150-2230 to 3020-3100 $\AA$ or 3020-3100 to 4150-4250 A rest frame, depending on the redshift of the quasar. Uncertainties in the spectral slopes were calculated from the error in the mean flux of each window (standard deviation across the window divided by the square root of the number of spectral resolution elements) and a wavelength uncertainty equal to half of the width of the window. Equivalent widths were measured for MgII and, for higher redshift objects, CIII], the only prominent emission lines in the spectra of these quasars.

Broadband optical magnitudes were obtained with the Steward Observatory 2.3 meter (nights of UT 1995 Feb $7 \&$ 8, Apr 8) and 1.5 meter (nights of UT 1995 Feb 19, Mar 14) telescopes. Exposure times were 5 minutes or less per frame. In almost all cases, each quasar was observed more than once per passband. Standard reduction techniques of bias subtraction and flat fielding were applied to all frames, followed by the determination of instrumental magnitudes for standard stars and program quasars using Stetson's (1987, 1990) DAOPHOT and DAOGROW packages. Additional data, flagged in Table 2, were drawn from a large multiband photometric study of the LBQS, currently in preparation.

The $R$ passband is roughly coincident with restframe $B$ at redshifts $z \sim 0.5$, making it more accurate to calculate $M_{B}$ from the $R$ magnitude, rather than observed $B$. This conversion can be expressed as

$$
M_{B}-R=-2.5\left[\log \left(\frac{\left(d_{L} / 10 \mathrm{pc}\right)^{2}}{(1+z)^{\alpha+1}}\right)+\log \left(\bar{F}_{B} / \bar{F}_{R}\right)+\log \left(f_{\nu}(R=0) / f_{\nu}(B=0)\right)\right],
$$

where $\bar{F}_{B}$ and $\bar{F}_{R}$ are the flux densities of a power law spectrum with index $\alpha$ averaged over the restframe $B$ and $R$ passbands, respectively, $d_{L}$ is the luminosity distance, and $f_{\nu}(B$ or $R=0)$ is the flux density for magnitude zero. The error in the conversion from $R$ to $M_{B}$ given an uncertainty in spectal index $\sigma_{\alpha}$, is

$$
\sigma_{\left(M_{B}-R\right)}=\frac{2.5 \sigma_{\alpha}}{\ln 10}\left[\ln (1+z)-\frac{\int \nu^{\alpha} \ln \nu B(\nu) d \nu}{\int \nu^{\alpha} B(\nu) d \nu}+\frac{\int \nu^{\alpha} \ln \nu R(\nu) d \nu}{\int \nu^{\alpha} R(\nu) d \nu}\right] .
$$


$B(\nu)$ and $R(\nu)$ are the filter transmission functions. The factor $\ln \nu$ arises from the derivative of $\nu^{\alpha}$ with respect to $\alpha$. Spectral indices determined for each quasar were used in these calculations. Absolute magnitude was derived from observed $V$ in an analogous manner for the two quasars in Table 2 without $R$ magnitudes. Uncertainties in the conversion from $V$ or $R$ to $M_{B}$ were typically $0.03 \mathrm{mag}$ and did not exceed $0.05 \mathrm{mag}$ for any object, leaving variability as the largest error component for the 13 quasars with new photometry. The final errors in $M_{B}$ listed for each object in Table 1, are a factor of 2 lower for the quasars with improved optical data, compared to other LBQS quasars at the same redshifts. Absolute magnitudes for LBQS quasars other than those listed in Table 2 were calculated using the method described in Paper II.

The newly obtained optical data, including one sigma errors, for selected radio-loud quasars are presented in Table 2 as follows:

Column (1): Object name.

Columns (2) through (4): $V$ magnitude, $B-V$ and $V-R$ colors, where available.

Column (5): Spectral index, $\alpha\left(f_{\nu} \propto \nu^{\alpha}\right)$.

Columns (6) and (7): Observed-frame equivalent widths for Mg II $\lambda 2798$ and CIII] $\lambda 1909$. 


\section{Distribution of Radio Luminosity and $\log R_{8.4}$ as Functions of $M_{B}$}

Figure 2 shows the distributions of $\log L_{8.4}$ and $\log R_{8.4}$ vs. $M_{B}$ for the data from Table 1 plus those from Table 2 in Paper II. Detections with $\log L_{8.4} \geq 25$ or alternatively $\log R_{8.4} \geq 1$ are considered to be radio loud, the same definitions employed in Paper II. Quasars in the new radio sample form a cluster of upper limits and detections with elevated radio luminosities in the magnitude range $-25<M_{B}<-23$, due to shorter integration times than the observations described in Paper II.

Radio-loud LBQS quasars are distributed fairly uniformly throughout a range of over two decades in $R_{8.4}$ and $L_{8.4}$ and across all absolute magnitudes sampled by the survey. No correlation between either $\log R_{8.4}$ or $\log L_{8.4}$ and $M_{B}$ is observed among the radio-loud quasars, consistent with previous studies by Miller et al. (1990) and Stocke et al. (1992). The apparent correlation between radio luminosity and absolute magnitude at low values of $\log L_{8.4}$ is the result of the LBQS flux limit.

Comparisons between the LBQS and other optically selected surveys, such as the PG, demonstrate the effects of different optical selection criteria on the inferred radio properties. Contrasting the LBQS with surveys selected at other wavebands, such as the X-ray selected EMSS, provides further insights into the relevant physical mechanisms and helps identify radio properties of quasars which are universal, regardless of selection frequency. The overall radio-loud $\left(\log L_{8.4}>25\right)$ fraction for the LBQS, 32/359 (9\%), is nearly identical to that in the EMSS, $39 / 411$ (9\%), but is less than half of the value for the PG, 24/114 (21\%). Overall fractions with $\log R_{8.4}>1$ are similar, with the LBQS at 31/359 (9\%), the EMSS at 46/411 (11\%), and the PG at 22/114 (19\%). Radio data for all of the PG quasars (Table 1 in Kellermann et al. 1989) and the EMSS detections classified as "AGN" and having radio fluxes (Table 4 in Stocke et al. 1991) are used in the comparisons throughout this paper. Radio fluxes and luminosities at other frequencies taken from the literature were extrapolated to $8.4 \mathrm{GHz}$ using a spectral index of -0.5 , the same value employed for the LBQS data. The radio luminosity and $\log R_{8.4}$ distributions vs. absolute magnitude for the LBQS differ from the PG and the EMSS, as shown in Figure 3. These plots were constructed by calculating the radio-loud fraction of objects within a 1 magnitude range centered on the $M_{B}$ value of each quasar in the sample, producing essentially a boxcar smoothing of the data. The error bars $( \pm 1 \sigma)$ shown at selected values of $M_{B}$ are the standard deviations of a fraction calculated from a binomial random variable: $\sigma=[f(1-f) / N]^{1 / 2}$, where $f$ is the radio-loud fraction in a 1.0 magnitude bin centered on $M_{B}$ containing $N$ points. The radio-loud fraction in the LBQS is constant at $\approx 10 \%$ for all but the most optically luminous quasars, rising to $\sim 35 \%\left(\sim 20 \%\right.$ for $\left.\log R_{8.4}=1\right)$ for $M_{B} \gtrsim-28$. The latter fraction is rather uncertain due to the small number of objects in this range.

A series of statistical tests were performed on the $M_{B}$ distributions of the radio-loud and radio-quiet quasars in the LBQS to quantify the significance of the observed trends. A Wilcoxon two-sample rank sum test indicated that the radio-loud and radio-quiet quasars do not have 
significantly different absolute magnitude distributions (the statistic differed by only $0.03 \sigma$ from the value expected under the null hypothesis of equivalent $M_{B}$ distributions) using the $\log R_{8.4}>1$ definition of radio-loud. There is some indication of a difference using the $\log L_{8.4}>25$ criterion (97\% confidence). However, when the 11 quasars with $M_{B}<-28$, the approximate region where the fraction of detections with $\log L_{8.4}>25$ is observed to rise, were excluded, no significant difference $(82 \%$ confidence level) was found between the radio-loud and radio-quiet distributions. The Wilcoxon test was also applied to the absolute magnitude distributions in a series of redshift bins in order to remove some of the degeneracy between $z$ and $M_{B}$ inherent in any flux-limited sample. Data for the 40 high-redshift quasars in Schmidt et al. (1995), discussed in more detail in Section 1 , were added to the LBQS radio sample to extend the redshift range. Two binning schemes were employed, one using the redshift ranges of Schmidt et al. (1995), shown in Figure 7, and in the other case the combined sample was divided into 5 bins of nearly equal numbers. The only moderately significant (96.8\% confidence) difference between the radio-loud, using either definition, and remaining distributions occured for $\log L_{8.4}>25$ in the equal-number bin covering the redshift range $2.34<z<4.90$. Again, this was due to the enhanced radio-loud fraction at bright absolute magnitudes. Ties in the rankings for the Wilcoxon tests were handled in the standard way by averaging the ranks of a group of quasars with the same $M_{B}$, and the probabilites quoted are for a two-sided test using the asymptotic normal distribution of the rank sum statistic.

The LBQS radio data, sorted by $M_{B}$, were divided into 10 bins each containing $\approx 36$ objects to perform some of the $\chi^{2}$ and binomial tests described in $\S 3.2 .2$ of Paper II. The results are very close to those in Paper II and are consistent with the results of the Wilcoxon tests above. The number of detections with $\log R_{8.4}>1$ in each bin is consistent with a constant radio-loud fraction equal to the average fraction for the whole LBQS radio sample (the probability that the sample is not consistent with the flat distribution is $81 \%$ ). This is not the case, however, using the radio luminosity criterion for radio-loud, which produces a distribution which is not flat at the $99.7 \%$ confidence level. The discrepency lies in the brightest absolute magnitude bin, $-28.7<M_{B}<-27.5$, and if this bin is excluded, the confidence level drops to an insignificant $76 \%$. The liklihood from a binomial distribution of selecting at least the observed number, 10/36 $(28 \%)$, of radio-loud quasars in the brightest bin, given the average radio-loud fraction for the full sample, is 0.00092. Since this bin was selected a posteriori because of its high incidence of radio-loud quasars, the true significance of the enhanced radio-loud fraction is the binomial probability multiplied by the number of bins (10), for a final confidence level of $99.1 \%$.

All of the statistical tests performed on the distribution of radio-loud objects with $M_{B}$ in the LBQS sample are consistent with the representation shown in Figure 3a: the radio-loud fraction is essentially constant except at the brightest absolute magnitudes, and even then it is only significantly higher using the $\log L_{8.4}>25$ criterion. Some possible interpretations of this distribution are discussed in Section 5 .

Both the PG and EMSS have substantially higher radio-loud fractions than the LBQS for $M_{B}$ brighter than -24 (see Figure 3, b \& c). Note that the PG has an anomolously high radio-loud 
fraction in this absolute magnitude range compared to other optically selected quasar samples (La Franca et al. 1994; Della Ceca et al. 1994). The reason for this discrepency remains unexplained. A strong trend in radio-loud fraction as a function of both absolute magnitude and redshift is expected in the EMSS, due to the well-known correlations between radio, X-ray, and optical luminosity (see, e.g., Worrall et al. 1987) and the X-ray flux limit. Figure 1 shows absolute magnitude plotted against redshift for the LBQS and EMSS, with the radio-loud $\left(\log L_{8.4}>25\right)$ quasars indicated by filled triangles. It is apparent that the observed trends with redshift and absolute magnitude are mostly degenerate. In particular, all but one of the radio-loud EMSS quasars brighter than $M_{B}=-24$ have $z>0.5$, whereas the bulk of the radio-quiet quasars fainter than this absolute magnitude are at redshifts $z<0.5$. However, some discrimination as a function of $M_{B}$ is possible in the redshift interval $0.4<z<0.7$. In this redshift range the radio-loud fraction is $7 / 20(35 \%)$ for $M_{B}<-24$ and $3 / 45(7 \%)$ among the fainter quasars, suggesting that at $z \sim 0.5$ the radio-loud fraction of X-ray selected quasars is dependent upon absolute magnitude, decreasing for $M_{B}$ fainter than -24 . The selection effect proposed by Peacock et al. (1986) does not explain the decline in radio-loud fraction for $M_{B}>-24$ in the EMSS, since the ratio of quasar to host galaxy optical luminosity is not expected to affect X-ray selection.

The Peacock et al. (1986) selection effect is still a viable explanation for the decrease in radio-loud fraction in the $\mathrm{PG}$, since a quantitative test of the applicability of this hypothesis to the PG cannot be performed, as was done for the LBQS in Paper II. Alternatively, the PG may have a nearly normal radio-loud fraction for $M_{B}>-24$, perhaps only slightly depressed by the selection effect, which only appears abnormally low because the fraction at brighter absolute magnitudes is atypically high. If the radio-loud fraction among the optically faint PG quasars is compared to the fraction at absolute magnitudes brighter than -24 in the LBQS, rather than in the PG, the significance of the change becomes equivocal. The fraction of quasars with $\log R_{8.4}>1$ in the faint PG sample $(2 / 45,4 \%)$ is not significantly different ( $71 \%$ confidence level) from the LBQS value of $23 / 286(8 \%)$ for $M_{B}<-24$. However, the difference is significant at the $98.6 \%$ confidence level using the $\log L_{8.4}$ definition of radio-loud, for which the fractions are 0/45 and 26/286 (9\%) in the PG and LBQS subsamples, respectively. If PG quasars with $M_{B}$ fainter than -22.7 , the faint absolute magnitude limit of the LBQS, are excluded from the comparison, the fractions for both definitions become 0/24, for which the confidence levels are $87 \%\left(\log R_{8.4}>1\right.$ ) and $90 \%$ $\left(\log L_{8.4}>25\right)$.

No pronounced decline in radio-loud fraction at absolute magnitudes fainter than -24 is seen in the LBQS, contrary to the other two samples (Figure 3). As noted in the introduction, a decrease was seen in this range in Paper II, but the number of objects with $M_{B}>-24$ was small (20), and the absolute magnitude uncertainties were \pm 0.4 mag. The new LBQS data increase the sample size at faint optical luminosities by more than a factor of 3 and decrease the magnitude uncertainties for radio-loud objects near $M_{B}=-24$ by a factor of 2 . Improved absolute magnitude determinations for two radio-loud quasars listed as slightly brighter than $M_{B}=-24$ in Paper II (see Figure 4 of that work) now place them fainter than this division, along with 4 new radio-loud 
quasars. The radio-loud $\left(\log L_{8.4}>25\right)$ fraction in the combined LBQS sample is $6 / 73(8 \%)$ at absolute magnitudes fainter than $M_{B}=-24$, nearly identical to the fraction $(26 / 286,9 \%)$ at brighter absolute magnitudes. Much of the discussion to date on the radio luminosity distribution of quasars at low optical luminosities has been based at least in part on the PG (Peacock et al. 1986; Miller et al. 1990; Padovani 1993; Falcke, Gopal-Krishna, \& Biermann 1995). The LBQS results show that the radio-loud fraction does not decline for $M_{B}>-24$ in all optically selected quasar samples. In cases where it does, the cause may be a selection effect at faint absolute magnitudes or an increased prevalence of radio-loud quasars at bright absolute magnitudes. 


\section{The Evolution of Radio and Optical Luminosity}

Radio-loud fraction is nearly constant in the LBQS over most of the redshift range of the survey, as shown in Figure 5. The smoothed fraction as a function of $z$ was calculated with a redshift smoothing interval of 0.5 in a manner analogous to its determination as a function of $M_{B}$ above. Some of the same statistical tests used in Section 3 were applied to the distributions of $\log L_{8.4}$ and $\log R_{8.4}$, with a similar result that the quantitative measures confirm the trends displayed graphically in Figure 5. The Wilcoxon rank sum statistic for detections with $\log R_{8.4}>1$ differs by only $0.2 \sigma$ from the value expected if these have the same redshift distribution as the remaining quasars in the LBQS radio sample. The radio-loud quasars have a moderately significantly (confidence level 95\%) different redshift distribution using $\log L_{8.4}>25$, but the significance disappears (revised confidence level 77\%) when the region with the observed spike in radio-loud fraction, $z>3$, is excluded. The results of a $\chi^{2}$ goodness of fit test of a model in which the radio-loud fraction is unevolving and equal to the sample average are not as clear-cut as those from the Wilcoxon test, because of a greater sensitivity to the rise in radio-loud fraction around $z \approx 1$. While the increase in radio-loud fraction for $z \approx 1$ is not nearly as great as the apparent climb at $z \geq 3$, this enhancement contributes significantly to the $\chi^{2}$ statistic. For example, the $\log R_{8.4}$ distribution, which has very close to the expected number of radio-loud quasars in the highest redshift bin, is inconsistent with a flat distribution at the $94 \%$ confidence level based on the $\chi^{2}$ test.

A model for the evolution of the radio-loud fraction was constructed from the radio luminosity functions of Dunlop \& Peacock (1990). They derived separate luminosity functions for flat and steep spectrum objects, divided at a spectral index $\alpha=-0.5$, from a radio-selected sample of quasars and radio galaxies. Distinct versions of the model were formed from the steep and flat spectrum RLF1 MEAN- $z$ luminosity functions, since radio spectral information is not available for most quasars in the LBQS. The luminosity functions were truncated at a radio luminosity equivalent to $\log L_{8.4}=28.85$, two decades below the limit employed by Dunlop \& Peacock (1990). The steep spectrum RLF1 function has an unphysical sharp upturn in number density at luminosities greater than this value. Function RLF2 remedies this with an imposed exponential cut-off at high luminosities, but the model based on this luminosity function does not match the observed data as well as the truncated RLF1. The true number of objects excluded by truncating the luminosity functions nearly 4 orders of magnitude above the radio-loud threshold of $\log L_{8.4}=25$ is expected to be relatively small and have negligible impact on the predicted space density of radio-loud quasars. The $\log R_{8.4}$ radio-loud criterion was also employed, although indirectly, as the luminosity functions do not provide a one-to-one correspondence between radio luminosity and absolute magnitude. A radio luminosity threshold which depends on redshift, $\log L_{8.4}=24.6+0.36 z$, was used to approximate the $\log R_{8.4}=1$ boundary (see Figure 7 in Paper II). The normalization of the predicted fraction is set by the overall radio-loud fraction of the LBQS, as described, along with other details of the model, in Paper II. The predicted evolution of radio-loud fraction for various model parameters is shown with the observed smoothed fraction in 
Figure 5. The flat spectrum versions match the data well, including the modest rise around $z=1$, at all except the highest redshifts, where the model does not reproduce the substantial rise in the fraction with $\log L_{8.4}>25$. A similar local maximum occurs in the steep spectrum version, but at higher redshifts than observed.

Many of the quasars at high redshift, where the radio-loud fraction rises, have absolute magnitudes $\sim-28$ (Figure 3a). It is not possible to disentangle redshift from $M_{B}$ as the causal variable for the enhanced radio-loud fraction using only the LBQS data. However, the radio-loud fraction in the restricted redshift $(1.8<z<2.5)$ sample of Miller et al. (1990) rises steeply for $M_{B}$ brighter than -27 , indicating that elevated radio-loud fraction at bright absolute magnitudes is a common feature of optically selected quasars over a wide range in redshift. A similar ambiguity between $M_{B}$ and $z$ involving the rise at $z=1$ was discussed in Paper II, where it was concluded that the effect is probably evolutionary. The new data support this, as there is no peak in radio-loud fraction at $M_{B} \sim-25.5$, the typical absolute magnitude for LBQS quasars with $z \sim 1$ (Figure B).

Optically selected quasars with $z>3.0$ have a radio-loud fraction similar to that of the LBQS. McMahon, Irwin, \& Hazard (1992) collected radio data for 29 quasars in the redshift range $3.5<z<4.7,4$ of which have $\log L_{8.4}>25$, and 3 have $\log R_{8.4}>1$. Individual absolute magnitudes and redshifts for these quasars were not published. Schneider et al. (1992) observed 22 quasars at $5 \mathrm{GHz}$, which were reobserved at $1.5 \mathrm{GHz}$ along with an additional 18 quasars by Schmidt et al. (1995). One target was detected in the original study, and it was also above the detection threshold at $1.5 \mathrm{GHz}$, along with two others which were not included in the first sample. The common detection has $\log L_{8.4}>25$ and $\log R_{8.4}>1$ for both observations. The remaining two low frequency detections have $\log L_{8.4}>25$, but only one is radio-loud by the $R_{8.4}$ criterion. The 40 quasars in the combined sample lie in a redshift interval $3.1<z<4.9$ and have absolute magnitudes ranging from -24.1 to -27.8 , with an average of -26.3 (values taken directly from Schmidt et al. 1995). Merging the high- $z$ data sets gives a high redshift radio-loud fraction of $7 / 69$ (10\%) for $\log L_{8.4}>25$ and $5 / 69$ (7\%) using the luminosity ratio criterion, values consistent with the LBQS in the redshift range $1.5<z<2.5$. A Wilcoxon test on the combined LBQS and Schmidt et al. (1995) samples found no statistically significant (88\% confidence level) difference in the redshift distributions of the detections with $\log L_{8.4}>25$ and the remaining quasars, even without removing the high- $z$ LBQS objects. Similar results were obtained when this combined sample was divided into 5 equal-number absolute magnitude bins. No significant differences in the redshift distributions were found among the bright bins, which contain the high- $z$ quasars. Ten of the quasars in the Schmidt et al. (1995) sample lie in a redshift range which overlaps the LBQS $(3.1<z<3.4)$, and none are radio loud. All of these have $M_{B}$ fainter than -27.3 , lending further support to the conclusion that the apparent rise in radio-loud fraction at high $z$ in the LBQS is a manifestation of a trend with absolute magnitude. Therefore, despite the appearance of Figure 5 at high redshift, there is substantial evidence that the radio-loud fraction of optically selected quasars does not evolve, aside from a modest peak around $z \approx 1$, over $65 \%$ of cosmic time, from 
$z=0.2$ to redshifts approaching 5: (1) the radio-loud fraction at high $z$ is consistent with that of the LBQS at moderate redshift; (2) a model which matches the LBQS data well at most redshifts does not reproduce the large fraction at high $z$; and (3) $M_{B}$ is the causal variable for the apparent rise in radio-loud fraction for $z \gtrsim 3$ in the LBQS, indicated by higher redshift samples. The overall radio-loud fraction in the high- $z$ sample and the LBQS data with $z<3.1$, together covering the redshift range $0.2<z<4.9$, is $37 / 426(9 \%)$ for $\log L_{8.4}>25$ and $34 / 426(8 \%)$ for $\log R_{8.4}>1$.

A nearly unevolving radio-loud fraction is inconsistent with many previous studies, which compared the predominantly low- $z$ PG with higher redshift optically selected samples and found a pronounced decrease in radio-loud fraction with increasing redshift (Peacock et al. 1986; Miller et al. 1990; Paper I; Schneider et al. 1992). La Franca et al. (1994) noted the same trend but found that evolution of the fraction was no longer indicated conclusively if the PG were excluded from the analysis. Recently, Schmidt et al. (1995) compared cumulative distributions of $\log R$ in 4 non-overlapping redshift ranges using data from a different survey for each distribution. The cumulative functions for the 3 highest redshift bins $(z>1)$ do not indicate substantial evolution over this range, but the low redshift distribution $(z<0.6)$, containing only PG quasars, has a significantly larger fraction of quasars with high values of $\log R$, especially for $M_{B}<-24.5$ (see their Figures $2 \& 3$ ). They concluded that the radio properties of quasars have evolved such that either the values of $R$ have decreased by two orders of magnitude from $z=0.3$ to 1.3 while maintaining the same distribution or that a population of quasars with very weak radio emission was more prevalent at $z>1$.

The redshift coverage of the LBQS is extensive enough to generate cumulative distributions in 3 of the 4 redshift bins from Schmidt et al. (1995) from a single well-defined survey, with the following constraints. The LBQS has little overlap with their highest redshift bin $(3.1<z<4.9)$, in which they used their own observations. Many of the PG quasars in the low- $z$ bin $(z<0.6)$ have $z<0.2$, the lower redshift limit of the LBQS. The absolute magnitude discrimination used for most of the analysis by Schmidt et al. (1995), $M_{B}<-24.5$, excludes almost $90 \%$ of these low- $z$ $\mathrm{PG}$ quasars but also restricts the LBQS to $z>0.35$. Redshift-segregated cumulative distributions of LBQS quasars are shown in Figure 0, along with the high- $z$ data of McMahon et al. (1992) and Schmidt et al. (1995) plotted as a single distribution. Kolmogorov-Smirnov tests were performed on these distributions in two ways. In one case, the true $\log R_{8.4}$ values of the upper limits were assumed to all lie below the smallest value for a detection, as displayed in Figure 7 . Only the portions of the distributions consisting of detections were compared to derive the test statistic. In the other case, the cumulative distributions were formed only from the detections, ignoring the upper limits completely. A significant difference was not found in any of these tests, the highest confidence level being $92 \%$. The cumulative distributions in the four redshift ranges are similar, indicating no evolution. In particular, the $z<0.6$ distribution, which had a cumulative fraction substantially higher than the other redshift ranges in the Schmidt et al. (1995) analysis, is slightly below that for $1.0<z<1.8$ for most values of $\log R_{8.4}$.

While the radio-loud fraction in the LBQS shows little dependence on redshift, the EMSS 
exhibits a rapid rise with redshift until reaching a plateau at $\approx 40 \%$ for $z>1$, as shown by the solid line in Figure 6. The fractions with $\log L_{8.4}>25$ and $\log R_{8.4}>1$ are nearly identical; the latter is slightly higher at low redshifts. Note that the behavior of the radio-loud fraction at the high redshift end of this plot is particularly uncertain, as there are only 4 quasars in the EMSS sample with $z>2$. This trend of radio-loud fraction with redshift is expected in X-ray selected samples as a result of the correlation between $\mathrm{X}$-ray and radio luminosities, which is thought to arise from a synchrotron self Compton component of the X-ray emission. At higher redshifts the flux limits of an X-ray survey correspond to higher luminosities, which, because of the correlation, tend to have higher radio luminosities.

A model for the relationship between radio-loud fraction and redshift in the EMSS was constructed using the expression for the dependence of X-ray luminosity on radio luminosity given by Brinkmann et al. (1995). They parameterized the correlation as $\log \left(l_{x}\right)=A_{\mathrm{rq}}+\beta_{\mathrm{rq}} \times \log \left(l_{r}\right)$, where $l_{x}$ and $l_{r}$ are X-ray and radio monochromatic luminosities at $2 \mathrm{keV}$ and $5 \mathrm{GHz}$, respectively. The constants were determined from ROSAT All-Sky Survey (Voges 1992) data on quasars in the 87 Green Bank radio survey (Condon et al. 1989) to be $A_{\mathrm{rq}}=8.44 \pm 0.40$, and $\beta_{\mathrm{rq}}=0.56 \pm 0.03$, where the uncertainties quoted here have been converted to $1 \sigma$ errors assuming Gaussian probability. Rest frame $2 \mathrm{keV}$ luminosities were determined from the survey flux limit at each redshift using a spectral index $\alpha=-1.13$ (Brinkmann et al. 1995). The corresponding value of $l_{r}$ was used as the lower luminosity limit in the integrals of the Dunlop \& Peacock (1990) steep and flat spectrum MEAN- $z$ RLF1 luminosity functions. The upper limit was the same as for the LBQS comparison model. These functions were also integrated over a redshift interval of 0.5 to find the total expected number of quasars. The number of radio-loud quasars was determined by integrating the same function using as the lower limit the larger of the radio-loud luminosity threshold, converted to the units employed in the luminosity function, or the luminosity determined from the correlation. This threshold was either $\log L_{8.4}=25$ or the redshift-dependent value equivalent to $\log R_{8.4}=1$ discussed earlier. The radio-loud boundary was not permitted to be lower than the luminosity corresponding to the $0.9 \mathrm{mJy}$ flux limit of the radio observations of the EMSS (Stocke et al. 1991). The EMSS did not have a uniform X-ray flux limit. A weighted average for each integral was calculated from 6 values of limiting X-ray flux determined from Gioia et al. (1990), with the weights proportional to the number of EMSS objects found at each limiting flux.

The predicted overall EMSS radio-loud fractions range mostly between $10 \%$ and $20 \%$ (cf. the observed fractions of $9 \%\left(\log L_{8.4}\right)$ and $11 \%\left(\log R_{8.4}\right)$ - Section 3$)$ as the values of $A_{\text {rq }}$ and $\beta_{\text {rq }}$ were varied within their $1 \sigma$ uncertainties. Values for these parameters were chosen so that the predicted average radio-loud fraction for the EMSS sample matched that observed. Each combination of radio-loud definition and luminosity function in the model was normalized separately. The predicted radio-loud fractions are plotted in Figure 6, along with the observed fractions. Most of the EMSS quasars are at low redshift (median $z$ is 0.268 ), where the radio-loud fraction is small, resulting in the modest overall radio-loud fraction. While there is little difference between 
the steep and flat spectrum models, the form of the predicted redshift dependence varies widely within the $1 \sigma$ uncertainties of the correlation parameters, and none of the model distributions match the observed one for $z>1$. The excess of predicted radio-loud quasars at high redshifts and the relative depletion at lower $z$ are directly connected, due to the normalization of the model by the observed average fraction. Radio luminosity has a spread of almost 2 dex for all $l_{x}$ in the Brinkmann et al. (1995) sample, which would lower the predicted radio-loud fraction, especially at high redshift. In particular, the model fraction would not reach $100 \%$ until a redshift at which the radio luminosity predicted from the X-ray limiting flux attained a value $\sim 1$ dex above the radio-loud boundary, since the intrinsic scatter in $l_{r}$ would provide some radio-quiet quasars up to this point. While these simple models do not conform to the observed data over all redshifts, they indicate that the rise in radio-loud fraction for $z<1$ is an expected feature of the X-ray/radio luminosity correlation. 


\section{Discussion}

Understanding the connection between the radio emission and the properties of the central engines of quasars continues to be an elusive problem. The constancy of the radio luminosity distribution over more than two orders of magnitude in optical luminosity and other similarities between radio-loud and radio-quiet quasars are somewhat suprising in the context of the standard black hole model of AGN. The radio emission mechanism appears to be only weakly tied to the mass accretion rate which presumably drives the optical and near-UV emission. Some models decouple the radio and optical emission, an important feature in attempting to explain the results from the LBQS and other studies which show little connection between these emission mechanisms. Coleman \& Dopita (1992) hypothesized that quasar radio luminosity may depend on the angle of the accretion disk with respect to the rotation axis of the central supermassive black hole. More recently however, Wilson \& Colbert (1995) proposed that the hole masses and accretion rates are similar in radio-loud and quiet quasars, which would account for the similarities in emission features at other wavebands. The total radio luminosity is presumed to derive from the spin energy of the hole via the Blandford-Znajek process (Blandford \& Znajek 1977). Powerful radio emission arises only from massive rapidly rotating holes, which in the view of Wilson \& Colbert are produced exclusively by mergers of two holes with similar large masses. They assume that accretion of material from the host galaxy is insufficient to spin up the hole to the necessary level because of either loss of angular momentum to gas around the hole or variable orientation of the accretion disk with respect to the spin axis of the hole. Therefore, two quasars with identical black hole masses and accretion rates can have vastly different radio luminosities depending on whether the hole was 1) built up by accretion and/or the merger of a large and small hole or 2) produced by the coalescence of two massive holes. The infrequency of mergers of galaxies containing massive black holes accounts for the rarity of powerful radio sources. The Wilson \& Colbert scenario is qualitatively consistent with the LBQS results that radio luminosity is not correlated with $M_{B}$ and that the radio-loud fraction is constant over most values of $M_{B}$.

A different radio emission mechanism which is correlated with optical luminosity may be responsible for the rise in radio-loud fraction at bright absolute magnitudes $\left(M_{B} \lesssim-28\right)$, seen also by Miller et al. (1990) and La Franca et al. (1994). Stocke et al. (1992) noted that radio and optical luminosity are correlated among the radio-quiet PG quasars. The detection frequency among the radio-quiet objects in the LBQS is too low to directly test for such a correlation, but there is indirect evidence that such a correlation is present: the fraction of radio detections among the radio-quiet LBQS quasars does not show a trend with absolute magnitude. If there were a null or negative correlation between the luminosities, the detection fraction among these objects would decline at higher redshifts, corresponding to brighter $M_{B}$, since the radio luminosity of the $3 \sigma$ detection limit increases with $z$. Stocke et al. (1992) hypothesized that the correlation arises from a component of radio emission powered by the kinetic energy of outflowing material driven by optical/UV radiation pressure. Radiation pressure and consequently outflow velocity aren't large enough to produce $\log L_{8.4}>25$ by this mechanism alone for $M_{B} \gtrsim-28$, but this 
emission component could exceed the radio-loud threshold $\left(\log L_{8.4}>25\right)$ at the brightest absolute magnitudes in the LBQS. In this scenario the enhanced radio-loud fraction at $M_{B} \lesssim-28$ arises from the combination of the black hole spin-driven component, which is independent of $M_{B}$, and the onset of powerful radio emission driven by outflows. The presence of two radio emission mechanisms, one correlated with optical luminosity, the other independent, accounts for the observed features of the radio luminosity distribution as a function of $M_{B}$ in the LBQS.

Neither radio emission mechanism explains the low radio-loud fraction observed at $M_{B}>-24$ in the EMSS and the PG surveys. Falcke et al. (1995) proposed that the decrease in radio-loud fraction at faint optical luminosities can be explained by a consolidation of two standard quasar unification schemes incorporating obscuring tori (e.g., Barthel 1989; Urry et al. 1991; Antonucci 1993). Radio-loud AGN with weaker (presumably fainter $M_{B}$ ) engines are observed as either BL Lac objects or FR I radio galaxies, not as quasars, as the opening angle of the torus is not wide enough in these objects for a direct view of the nuclear region free from contamination by the jet emission. This explanation is not directly tenable for the LBQS, since the incidence of radio-loud quasars is not observed to change at faint $M_{B}$. It is possible that, due to some poorly understood difference between the PG and the LBQS, orientation effects are present in the LBQS at a weaker level than originally proposed by Falcke et al. (1995) to explain the PG results. If the lines of sight to the radio-loud quasars with $M_{B}>-24$ lie close to relativistic jets (to avoid obscuration by the tori), the emission lines are expected to have low equivalent widths due to their dilution by doppler-boosted optical synchrotron emission; i.e., these AGN would be BL Lac-like, but not so much that they would be classified as BL Lacs. However, the radio-loud LBQS quasars with faint absolute magnitudes are not close to being classified as BL Lacs, as 4/6 have prominent emission lines, and the lines in the remaining two, while weaker, still have MgII equivalent widths of approximately $15 \AA$ (Table 2). AGN are classified as BL Lacs typically when they have emission line equivalent widths $<5 \AA$ (e.g., Stocke et al. 1991). The model does not appear to be applicable to optically selected quasar samples, as the proposed orientation effect is not present in the LBQS at a detectable level, and a reasonable alternative explanation exists for the $\mathrm{PG}$, although the model may partially account for the decrease observed in the EMSS. 


\section{Summary}

i. The distribution of radio luminosity does not depend on absolute magnitude over most of the range of $M_{B}$ in the LBQS. The radio-loud fraction remains constant at $\approx 10 \%$ for $-28 \lesssim M_{B} \lesssim-23$ but rises at brighter absolute magnitudes to $(20 \pm 10) \%$ for $\log R_{8.4}>1$ and $(35 \pm 15) \%$ for $\log L_{8.4}>25$.

ii. Radio-loud fraction is nearly unevolving at a value of $\approx 10 \%$ in the LBQS and three high- $z$ optically selected samples, which together span a redshift range from $z=0.2$ to redshifts approaching 5 .

iii. The PG survey differs significantly from several optically selected samples. The sudden decrease in radio-loud fraction for $M_{B}$ fainter than -24 observed in the PG is not present in the expanded LBQS sample. The fraction of quasars with $\log R_{8.4}>1$ in the PG for $M_{B}>-24$ is not significantly different from the LBQS fraction, but the PG has a much greater percentage of radio-loud quasars at brighter absolute magnitudes. High radio-loud fraction in the PG relative to other optically selected samples has led several investigators to conclude that the fraction evolves to lower values at higher $z$. This trend is not seen in the LBQS, which provides a single well-defined sample with wide redshift coverage $(0.2<z<3.4)$. The anomolously high radio-loud fraction in the PG sample remains unexplained.

iv. The radio properties of the X-ray selected EMSS also differ from those of the LBQS. The rapid rise in radio-loud fraction observed for $M_{B} \approx-24$ arises primarily from a well-established correlation between X-ray and radio luminosity. However, there is still a dependence of radio-loud fraction on $M_{B}$ in the redshift range $0.4<z<0.7$, an interval containing substantial numbers of quasars both brighter and fainter than $M_{B}=-24$.

v. The behavior of the radio properties of the LBQS quasars as a function of $M_{B}$ is consistent with the existence of two radio emission mechanisms, one correlated with optical luminosity, the other independent.

The authors thank Pat Hall for conducting some observations and Steve Warren for helpful discussions. The data would not have been obtained without the capable and friendly help of the operations staffs at the VLA, MMT, and Steward Observatory. Commentary from an anonymous referee led to clarification of the manuscript. This work was partially supported by NSF grant AST 93-20715 and NASA grant NGT-51152, a NASA Graduate Student Researchers Program Fellowship (EJH). This research has made use of the NASA Astrophysics Data System (ADS). 


\section{REFERENCES}

Antonucci, R. 1993, ARA\&A, 31, 473

Bahcall, J. N., Kirhakos, S., \& Schneider, D. P. 1996, ApJ, 457, 557

Barthel, P. D. 1989, ApJ, 336, 606

Blandford, R. D., \& Znajek, R. L. 1977, MNRAS, 179, 433

Brinkmann, W., Siebert, J., Reich, W., Fürst, E., Reich, P., Voges, W., Trümper, J., Wielebinski, R. 1995, A\&AS, 109, 147

Coleman, C. S., \& Dopita, M. A. 1992, Proc. Astron. Soc. Australia, 10, 107

Condon, J. J., O’Dell, S. L., Puschell, J. J., \& Stein, W. A. 1981, ApJ, 246, 624

Condon, J. J., Broderick, J. J., Seielstad, G. A. 1989, AJ, 97, 1064

Della Ceca, R., Zamorani, G., Maccacaro, T., Wolter, A., Griffiths, R., Stocke, J. T., \& Setti, G. 1994, ApJ, 430, 533

Disney, M. J., et al. 1995, Nature, 376, 150

Dunlop, J. S., \& Peacock, J. A. 1990, MNRAS, 247, 19

Falcke, H., Gopal-Krishna, Biermann, P. L. 1995, A\&A, 298, 395

Francis, P. J. 1993, ApJ, 407, 519

Francis, P. J., Hewett, P. C., Foltz, C. B., Chaffee, F. H., Weymann, R. J., \& Morris, S. L. 1991, ApJ, 373, 465

Gioia, I. M., Maccacaro, T., Schild, R. E., Wolter, A., Stocke, J. T., Morris, S. L., \& Henry, J. P. 1990, ApJS, 72, 567

Hewett, P. C., Foltz, C. B., \& Chaffee, F. H. 1995, AJ, 109, 1498

Hooper, E. J., Impey, C. D., Foltz, C. B., \& Hewett, P. C. 1995, ApJ, 445, 62 (Paper II)

Hutchings, J. B., Holtzman, J., Sparks, W. B., Morris, S. C., Hanisch, R. J., \& Mo, J. 1994, ApJ, 429, L1

Kellermann, K. I., Sramek, R., Schmidt, M., Shaffer, D. B., \& Green, R. 1989, AJ, 98, 1195 (PG)

La Franca, F., Gregorini, L., Cristiani, S., De Ruiter, H., \& Owen, F. 1994, AJ, 108, 1548

Marshall, H. L. 1987, ApJ, 316, 84

McMahon, R. G., Irwin, M. J., \& Hazard, C. 1992, in X-ray Emission from Active Galactic Nuclei and the Cosmic X-ray Background, ed. W. Brinkmann \& J. Trümper (Garching: Max-Planck-Institut für extraterrestrische Physik), 399

Miller, L., Peacock, J. A., \& Mead, A. R. G. 1990, MNRAS, 244, 207

Padovani, P. 1993, MNRAS, 263, 461

Peacock, J. A., Miller, L., \& Longair, M. S. 1986, MNRAS, 218, 265 
Rees, M. J. 1984, ARA\&A, 22, 471

Sandage, A. 1965, ApJ, 141, 1560

Schmidt, M., van Gorkom, J. H., Schneider, D. P., \& Gunn, J. E. 1995, AJ, 109, 473

Schneider, D. P., van Gorkom, J. H., Schmidt, M., \& Gunn, J. E. 1992, AJ, 103, 1451

Smith, E. P., Heckman, T. M., Bothun, G. D., Romanishin, W., \& Balick, B. 1986, ApJ, 306, 64

Sramek, R. A., \& Weedman, D. W. 1980, ApJ, 238, 435

Stetson, P. B. 1987, PASP, 99, 191

Stetson, P. B. 1990, PASP, 102, 932

Stocke, J. T., Morris, S. L., Gioia, I. M., Maccacaro, T., Schild, R., Wolter, A., Fleming, T. A., \& Henry, J. P. 1991, ApJS, 76, 813

Stocke, J. T., Morris, S. L., Weymann, R. J., \& Foltz, C. B. 1992, ApJ, 396, 487

Urry, C. M., Padovani, P., \& Stickel, M. 1991, ApJ, 382, 501

Véron-Cetty, M. P., \& Woltjer, L. 1990, A\&A, 236, 69

Visnovsky, K. L., Impey, C. D., Foltz, C. B., Hewett, P. C., Weymann, R. J., \& Morris, S. L. 1992, ApJ, 391, 560 (Paper I)

Voges, W. 1992, in Proceedings of the European International Space Year Conference "Space sciences with particular emphasis on high-energy astrophysics," ESA ISY-3, ed. T. D. Guyenne \& J. J. Hunt (Paris: ESA Publications), 9

Wilson, A. S., \& Colbert, E. J. M. 1995, ApJ, 438, 62

Worrall, D. M., Giommi, P., Tananbaum, H., \& Zamorani, G. 1987, ApJ, 313, 596 


\section{Figure Captions}

Fig. 1 Distributions of $M_{B}$ against redshift for the LBQS radio detections (filled circles) and upper limits (horizontal lines). Y-shaped symbols represent LBQS quasars without radio observations. All of the latter class with $z>2.5$ are plotted, but the large numbers at lower redshifts would over-clutter the plot. Therefore, a random selection of $1 / 3$ of the quasars with $z<2.5$ lacking radio data are plotted.

Fig. 2 The distribution of (a) $\log R_{8.4}$ and (b) $\log L_{8.4}$ against $M_{B}$ for the LBQS. The data shown consist of the new observations from Table 1 and those from Table 2 in Paper II. Filled circles represent detections, and horizontal lines are upper limits. Error bars $( \pm 1 \sigma)$, calculated as described in Section 2, are shown for a representative point at bright $\left(M_{B}=-27.8 ; \log R_{8.4}=3.5\right.$, $\left.\log L_{8.4}=28.3\right)$ and and one at faint $\left(M_{B}=-23.2 ; \log R_{8.4}=1.3, \log L_{8.4}=24.3\right)$ absolute magnitudes and individually for those quasars which were reobserved in the optical. Error bars in $\log L_{8.4}$ are not plotted in (b) for the quasars with new photometry to avoid unnecessary crowding and because the radio luminosity uncertainties are not affected by the new data.

Fig. 3 Smoothed radio-loud fraction as a function of $M_{B}$ (smoothed over an interval of 1.0 mag) for the LBQS (a), the PG (b), and the EMSS (c) surveys. Solid lines represent the fraction with $\log L_{8.4}>25$, and dotted lines are for $\log R_{8.4}>1$. Error bars, calculated as described in the text, are shown at selected values of $M_{B}$.

Fig. (1) Absolute magnitude vs. redshift for the LBQS (a) and the EMSS (b). This is similar to Figure 1, but in this case the filled triangles are radio-loud ( $\log L_{8.4}>25$ ) detections, crosses represent radio-quiet detections, and horizontal lines indicate upper limits, assumed to be radio-quiet.

Fig. 5 Smoothed radio-loud fraction as a function of redshift in the LBQS sample using the (a) $\log L_{8.4}>25$ and (b) $\log R_{8.4}>1$ criteria. Solid lines indicate the observed data, while dotted and dashed lines represent the flat and steep spectrum model predictions, respectively. Values for the data as well as the models were calculated with a redshift smoothing interval of 0.5. Error bars, calculated in an analogous manner to those in Fig. 3, are shown at representative data values.

Fig. 6 Smoothed radio-loud fraction as a function of redshift in the EMSS sample using the (a) $\log L_{8.4}>25$ and (b) $\log R_{8.4}>1$ criteria. Solid lines indicate the observed data, while dotted and dashed lines represent the flat and steep spectrum model predictions, respectively. Values for the data as well as the models were calculated with a redshift smoothing interval of 0.5. The error bars are analogous to those in the other plots of smoothed radio-loud fraction.

Fig. 7 The cumulative distributions $G\left(>\log R_{8.4}\right)$ of LBQS quasars in three redshift ranges: squares represent $z<0.6$; filled circles $1.0<z<1.8$; and plus signs $1.8<z<2.5$. The high redshift range $3.1<z<4.9$, represented by stars, contains the samples of McMahon et al. (1992) and Schmidt et al. (1995). Panel (a) includes all quasars with $M_{B}<-23$, and the absolute 
magnitude restriction is $M_{B}<-24.5$ in panel (b). The redshift bins, symbols, and absolute magnitude ranges match those used in Schmidt et al. (1995). Only the detections are plotted for clarity. All of the quasars in each redshift range were used to calculate the cumulative probabilities, assuming that all of the upper limits have $\log R_{8.4}$ values less than the lowest detection. 
Table 1:

Table 2:

Fig. 1.-

Fig. 2.-

Fig. 3.-

Fig. 4.-

Fig. 5.-

Fig. 6.-

Fig. 7.- 


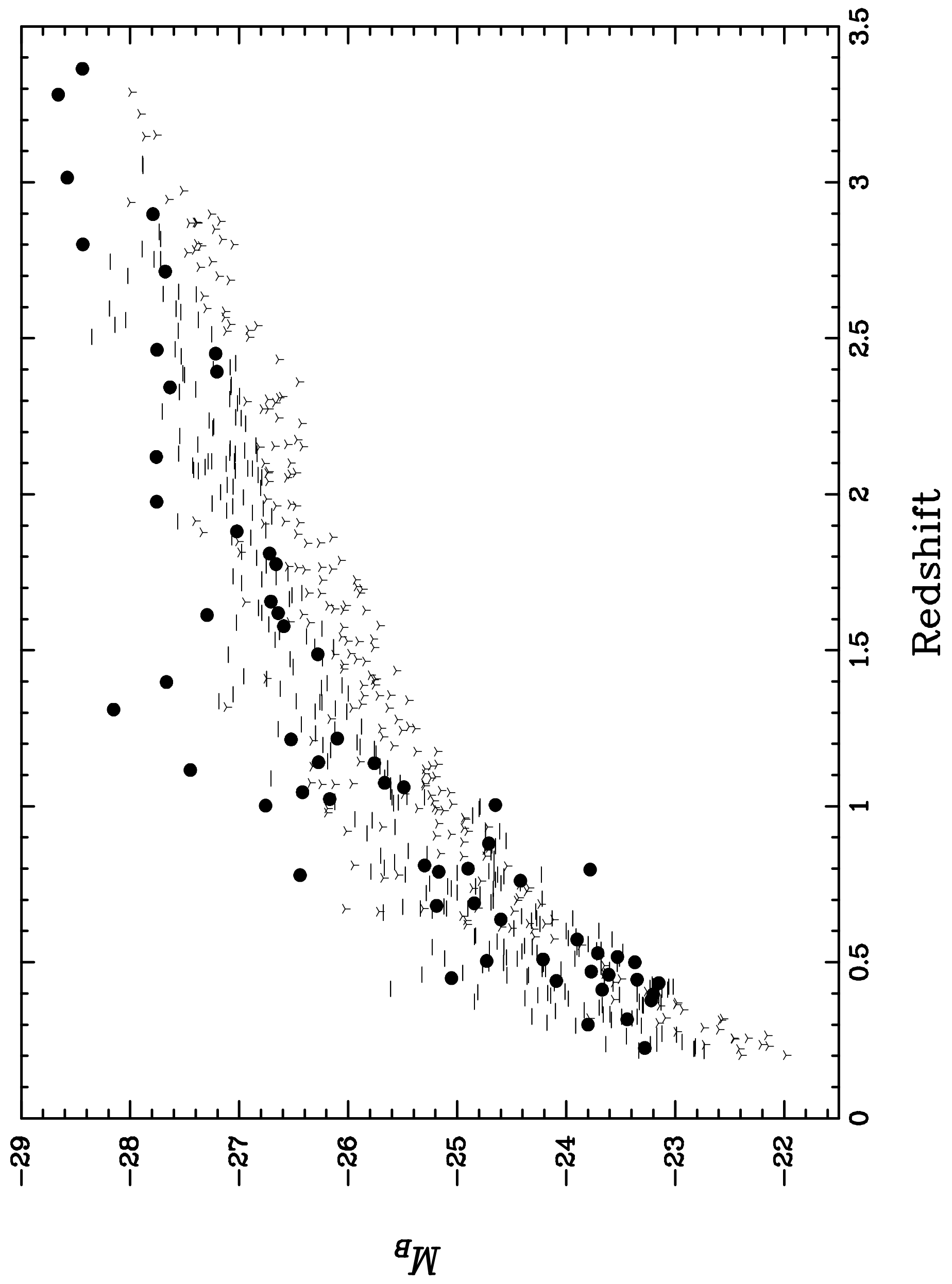


TABLE 1

Radio and Optical Observations of LBQS QUASARS

\begin{tabular}{|c|c|c|c|c|c|c|}
\hline $\begin{array}{c}\text { Source } \\
(1)\end{array}$ & $\begin{array}{c}B \\
(2)\end{array}$ & $\begin{array}{c}z \\
(3)\end{array}$ & $\begin{array}{l}M_{B} \\
(4)\end{array}$ & $\begin{array}{c}S_{8.4} \\
(\mathrm{mJy}) \\
(5)\end{array}$ & $\begin{array}{l}\log R_{8.4} \\
\quad(6)\end{array}$ & $\begin{array}{c}\log L_{8.4} \\
\left(\mathrm{WHz}^{-1}\right) \\
(7)\end{array}$ \\
\hline $0002-0243$ & 18.30 & 0.432 & $-23.57 \pm 0.40$ & $<0.41$ & 0.38 & $<23.52$ \\
\hline $0004+0036$ & 17.80 & 0.317 & $-23.44 \pm 0.38$ & $0.84 \pm 0.13$ & $0.47 \pm 0.17$ & $23.56 \pm 0.08$ \\
\hline $0004+0224$ & 17.34 & 0.300 & $-23.80 \pm 0.37$ & $1.31 \pm 0.13$ & $0.48 \pm 0.16$ & $23.70 \pm 0.06$ \\
\hline $0006+0015$ & 17.42 & 0.263 & $-23.45 \pm 0.37$ & $<0.42$ & $<0.00$ & $<23.09$ \\
\hline $0010+0035$ & 17.85 & 0.363 & $-23.67 \pm 0.39$ & $<0.78$ & $<\quad 0.47$ & $<23.65$ \\
\hline $0010+0131$ & 18.73 & 0.433 & $-23.15 \pm 0.41$ & $2.32 \pm 0.16$ & $1.30 \pm 0.18$ & $24.27 \pm 0.07$ \\
\hline $0017+0209$ & 17.54 & 0.401 & $-24.17 \pm 0.38$ & $<0.38$ & $<0.04$ & $<23.41$ \\
\hline $0020+0018$ & 18.62 & 0.423 & $-23.20 \pm 0.41$ & $<0.41$ & 0.51 & $<23.50$ \\
\hline $0021+0241$ & 17.94 & 0.495 & $-24.21 \pm 0.40$ & $<0.42$ & 0.25 & $<23.64$ \\
\hline $0021-0301$ & 18.79 & 0.422 & $-23.02 \pm 0.41$ & $<0.41$ & $<0.58$ & $<23.50$ \\
\hline $0022+0015$ & 16.92 & 0.404 & $-24.81 \pm 0.37$ & $<0.41$ & $<-0.18$ & $<23.45$ \\
\hline $0028-0101$ & 18.45 & 0.543 & $-23.88 \pm 0.42$ & $<0.48$ & $<\quad 0.52$ & $<23.78$ \\
\hline $0042-2550$ & 18.32 & 0.454 & $-23.65 \pm 0.40$ & $<0.42$ & 0.40 & $<23.57$ \\
\hline $0047-2647$ & 18.62 & 0.495 & $-23.53 \pm 0.41$ & $<0.44$ & 0.54 & $<23.66$ \\
\hline $0049-2840$ & 18.74 & 0.640 & $-23.94 \pm 0.44$ & $<0.51$ & 0.67 & $<23.95$ \\
\hline $0050-2742$ & 17.89 & 0.479 & $-24.19 \pm 0.39$ & $<0.43$ & 0.24 & $<23.62$ \\
\hline $0052-0203$ & 18.48 & 0.577 & $-23.98 \pm 0.43$ & $<0.40$ & $<\quad 0.46$ & $<23.75$ \\
\hline $0053+0124$ & 17.82 & 0.440 & $-24.09 \pm 0.39$ & $4.78 \pm 0.16$ & $1.25 \pm 0.17$ & $24.60 \pm 0.06$ \\
\hline $0055-0206$ & 17.50 & 0.239 & $-23.17 \pm 0.38$ & $<0.44$ & $<0.05$ & $<23.03$ \\
\hline $0057-0135$ & 17.94 & 0.325 & $-23.35 \pm 0.39$ & $<0.43$ & 0.24 & $<23.29$ \\
\hline $0058+0205$ & 18.54 & 0.600 & $-24.00 \pm 0.44$ & $<0.39$ & 0.46 & $<23.77$ \\
\hline $0059-2525$ & 17.61 & 0.454 & $-24.36 \pm 0.38$ & $<0.47$ & 0.16 & $<23.61$ \\
\hline $0100+0205$ & 17.53 & 0.393 & $-24.14 \pm 0.38$ & $<0.43$ & 0.08 & $<23.45$ \\
\hline $0101+0009$ & 17.42 & 0.394 & $-24.26 \pm 0.38$ & $<0.40$ & 0.01 & $<23.42$ \\
\hline $0110-0047$ & 18.70 & 0.412 & $-23.07 \pm 0.41$ & $<0.43$ & 0.56 & $<23.49$ \\
\hline $0251-0054$ & 17.77 & 0.433 & $-24.11 \pm 0.39$ & $<0.45$ & 0.21 & $<23.56$ \\
\hline $1137+0110^{\mathrm{II}}$ & 18.24 & 1.138 & $-25.76 \pm 0.21$ & $70.19 \pm 2.37$ & $2.57 \pm 0.15$ & $26.57 \pm 0.13$ \\
\hline $1138+0003^{\text {II }}$ & $18.35^{\mathrm{m}}$ & 0.500 & $-23.37 \pm 0.25$ & $37.73 \pm 0.81$ & $2.55 \pm 0.12$ & $25.60 \pm 0.07$ \\
\hline $1148-0033^{\text {II }}$ & 17.73 & 0.800 & $-24.90 \pm 0.25$ & $4.14 \pm 0.12$ & $1.38 \pm 0.14$ & $25.04 \pm 0.10$ \\
\hline $1209+1259$ & 18.57 & 0.418 & $-23.23 \pm 0.42$ & $<0.44$ & $<0.52$ & $<23.52$ \\
\hline $1210+1434$ & 18.62 & 0.870 & $-24.75 \pm 0.50$ & $<0.38$ & 0.48 & $<24.09$ \\
\hline $1211+0848^{\mathrm{II}}$ & 17.84 & 0.810 & $-25.30 \pm 0.19$ & $7.06 \pm 0.15$ & $1.46 \pm 0.13$ & $25.29 \pm 0.10$ \\
\hline $1212+0945$ & 18.24 & 0.373 & $-23.33 \pm 0.41$ & $<0.43$ & $<0.36$ & $<23.40$ \\
\hline $1212+1217$ & 18.72 & 0.872 & $-24.65 \pm 0.51$ & $<0.40$ & $<0.54$ & $<24.11$ \\
\hline $1218+1734$ & $18.36^{\mathrm{m}}$ & 0.444 & $-23.35 \pm 0.23$ & $25.36 \pm 1.11$ & $2.28 \pm 0.12$ & $25.33 \pm 0.07$ \\
\hline $1222+1010$ & 18.35 & 0.398 & $-23.35 \pm 0.41$ & $<0.42$ & $<0.41$ & $<23.46$ \\
\hline $1222+1235^{\mathrm{II}}$ & $17.93^{\mathrm{m}}$ & 0.412 & $-23.67 \pm 0.26$ & $21.80 \pm 0.43$ & $2.02 \pm 0.12$ & $25.19 \pm 0.06$ \\
\hline $1223+1226$ & 18.70 & 0.874 & $-24.68 \pm 0.51$ & $<0.42$ & $<0.55$ & $<24.13$ \\
\hline $1224+1604$ & 18.81 & 0.533 & $-23.48 \pm 0.44$ & $<0.49$ & $<0.68$ & $<23.78$ \\
\hline $1228+1001$ & 18.07 & 0.637 & $-24.60 \pm 0.44$ & $2.81 \pm 0.22$ & $1.14 \pm 0.20$ & $24.68 \pm 0.09$ \\
\hline $1228+1411$ & 18.70 & 0.512 & $-23.51 \pm 0.44$ & $<0.53$ & $<0.66$ & $<23.77$ \\
\hline $1229+0834$ & 18.65 & 0.761 & $-24.42 \pm 0.48$ & $1.06 \pm 0.26$ & $0.94 \pm 0.24$ & $24.41 \pm 0.15$ \\
\hline $1229-0207$ II & 17.69 & 1.045 & $-26.42 \pm 0.18$ & $283.9 \pm 23.9$ & $2.84 \pm 0.14$ & $27.11 \pm 0.12$ \\
\hline $1230+1427$ & 18.75 & 0.422 & $-23.06 \pm 0.43$ & $<0.42$ & $<0.57$ & $<23.51$ \\
\hline $1230-0015^{\mathrm{II}}$ & 17.04 & 0.470 & $-23.77 \pm 0.28$ & $63.33 \pm 1.91$ & $2.56 \pm 0.13$ & $25.77 \pm 0.06$ \\
\hline $1230+1627 \mathrm{~A}$ & 18.74 & 0.918 & $-24.74 \pm 0.52$ & $<0.49$ & $<0.63$ & $<24.24$ \\
\hline
\end{tabular}


TABLE 1-Continued

\begin{tabular}{|c|c|c|c|c|c|c|}
\hline $\begin{array}{l}\text { Source } \\
\text { (1) }\end{array}$ & $\begin{array}{c}B \\
(2)\end{array}$ & $\begin{array}{c}z \\
(3)\end{array}$ & $\begin{array}{l}M_{B} \\
(4)\end{array}$ & $\begin{array}{c}S_{8.4} \\
(\mathrm{mJy}) \\
(5)\end{array}$ & $\begin{array}{l}\log R_{8.4} \\
\quad(6)\end{array}$ & $\begin{array}{c}\log L_{8.4} \\
\left(\mathrm{WHz}^{-1}\right) \\
(7)\end{array}$ \\
\hline $1231+1627$ & 18.85 & 0.999 & $-24.79 \pm 0.54$ & $<0.47$ & 0.67 & $<24.29$ \\
\hline $1231+1728$ & 18.38 & 0.623 & $-24.24 \pm 0.45$ & $<0.41$ & 0.42 & $<23.83$ \\
\hline $1232+1433$ & 18.88 & 0.920 & $-24.61 \pm 0.52$ & $<0.37$ & 0.57 & $<24.12$ \\
\hline $1234+1217$ & 18.48 & 0.662 & $-24.28 \pm 0.46$ & $<0.44$ & 0.49 & $<23.91$ \\
\hline $1236+1802$ & 18.70 & 0.517 & $-23.53 \pm 0.44$ & $1.12 \pm 0.12$ & $0.99 \pm 0.19$ & $24.11 \pm 0.09$ \\
\hline $1237+0824$ & 18.65 & 0.412 & $-23.12 \pm 0.42$ & $<0.53$ & $<0.63$ & $<23.58$ \\
\hline $1237+1100$ & 18.69 & 0.881 & $-24.71 \pm 0.51$ & $0.70 \pm 0.13$ & $0.77 \pm 0.24$ & $24.36 \pm 0.14$ \\
\hline $1238+1401$ & 18.41 & 0.641 & $-24.27 \pm 0.45$ & $<0.45$ & $<0.47$ & $<23.89$ \\
\hline $1240+1551$ & 18.86 & 0.573 & $-23.58 \pm 0.45$ & $<0.36$ & 0.56 & $<23.70$ \\
\hline $1240+1746$ & 18.05 & 0.549 & $-24.31 \pm 0.43$ & $<0.42$ & 0.30 & $<23.73$ \\
\hline $1241+0107$ & 18.49 & 0.786 & $-24.66 \pm 0.48$ & $<0.45$ & 0.50 & $<24.07$ \\
\hline $1241+0844$ & 18.37 & 0.378 & $-23.22 \pm 0.41$ & $0.54 \pm 0.14$ & $0.52 \pm 0.21$ & $23.52 \pm 0.12$ \\
\hline $1242+1719$ & 18.64 & 0.540 & $-23.68 \pm 0.44$ & $<0.44$ & $<0.56$ & $<23.74$ \\
\hline $1244+1358$ & 18.84 & 0.601 & $-23.70 \pm 0.45$ & $<0.39$ & 0.59 & $<23.78$ \\
\hline $1244+1529$ & 18.90 & 0.781 & $-24.23 \pm 0.49$ & $<0.42$ & 0.64 & $<24.04$ \\
\hline $1250+0109$ & 18.45 & 0.792 & $-24.71 \pm 0.48$ & $<0.39$ & 0.43 & $<24.02$ \\
\hline $1308+0047$ & 18.63 & 0.428 & $-23.21 \pm 0.40$ & $<0.69$ & $<0.74$ & $<23.73$ \\
\hline $1308-0111$ & 18.51 & 1.004 & $-24.65 \pm 0.18$ & $4.58 \pm 0.21$ & $1.72 \pm 0.14$ & $25.28 \pm 0.12$ \\
\hline $1311+0131$ & 18.50 & 0.781 & $-24.63 \pm 0.47$ & $<0.42$ & $<0.47$ & $<24.03$ \\
\hline $1313+0020$ & 18.32 & 0.735 & $-24.67 \pm 0.45$ & $<0.41$ & 0.39 & $<23.97$ \\
\hline $1313-0228$ & 18.67 & 0.704 & $-24.22 \pm 0.45$ & $<0.30$ & 0.41 & $<23.80$ \\
\hline $1315+0150$ & 18.60 & 0.539 & $-23.72 \pm 0.41$ & $<0.37$ & 0.47 & $<23.66$ \\
\hline $1316+0103$ & 18.53 & 0.394 & $-23.15 \pm 0.39$ & $<0.41$ & 0.47 & $<23.44$ \\
\hline $1321-0213$ & 18.83 & 0.991 & $-24.80 \pm 0.52$ & $<0.36$ & $<0.54$ & $<24.17$ \\
\hline $1322-0204$ & 18.54 & 0.573 & $-23.90 \pm 0.42$ & $0.53 \pm 0.14$ & $0.60 \pm 0.22$ & $23.87 \pm 0.14$ \\
\hline $1323+0205$ & 18.55 & 0.641 & $-24.13 \pm 0.43$ & $<0.51$ & $<0.59$ & $<23.95$ \\
\hline $1324+0125$ & 18.73 & 0.970 & $-24.86 \pm 0.52$ & $<0.48$ & 0.63 & $<24.28$ \\
\hline $1326-0257$ & 18.19 & 0.743 & $-24.83 \pm 0.45$ & $<0.41$ & 0.34 & $<23.98$ \\
\hline $1328+0205$ & 18.16 & 0.692 & $-24.69 \pm 0.44$ & $<0.41$ & 0.33 & $<23.92$ \\
\hline $1330-0156$ & 18.87 & 0.889 & $-24.55 \pm 0.50$ & $<0.48$ & 0.67 & $<24.20$ \\
\hline $1334+0053$ & 18.29 & 0.647 & $-24.41 \pm 0.44$ & $<1.76$ & 1.02 & $<24.49$ \\
\hline $1335-0241$ & 18.09 & 0.608 & $-24.48 \pm 0.43$ & $<0.38$ & 0.27 & $<23.77$ \\
\hline $1340-0020$ & 18.15 & 0.786 & $-25.00 \pm 0.47$ & $<0.40$ & 0.31 & $<24.02$ \\
\hline $1344-0227$ & 18.29 & 0.511 & $-23.92 \pm 0.41$ & $<0.43$ & 0.40 & $<23.68$ \\
\hline $1345-0000$ & 17.99 & 0.552 & $-24.38 \pm 0.41$ & $<0.45$ & 0.31 & $<23.77$ \\
\hline $1350+0052$ & 18.43 & 0.485 & $-23.68 \pm 0.41$ & $<0.45$ & 0.47 & $<23.65$ \\
\hline $1429-0039$ & 18.21 & 0.362 & $-23.30 \pm 0.40$ & $<0.40$ & 0.33 & $<23.35$ \\
\hline $1429-0246$ & 18.63 & 0.853 & $-24.69 \pm 0.50$ & $<0.40$ & 0.50 & $<24.09$ \\
\hline $1430-0046^{\mathrm{II}}$ & 17.57 & 1.023 & $-26.17 \pm 0.17$ & $14.97 \pm 0.31$ & $1.64 \pm 0.14$ & $25.81 \pm 0.12$ \\
\hline $1433+0011$ & 18.57 & 0.583 & $-23.91 \pm 0.44$ & $<0.39$ & $<0.48$ & $<23.75$ \\
\hline $1433-0016$ & 17.80 & 0.325 & $-23.49 \pm 0.39$ & $<0.47$ & 0.22 & $<23.32$ \\
\hline $1435+0130$ & 18.33 & 0.633 & $-24.32 \pm 0.45$ & $<0.41$ & 0.41 & $<23.84$ \\
\hline $1437-0143$ & 18.15 & 0.718 & $-24.79 \pm 0.46$ & $<0.49$ & 0.40 & $<24.03$ \\
\hline $1438+0210$ & 19.34 & 0.797 & $-23.78 \pm 0.20$ & $236.6 \pm 11.0$ & $3.58 \pm 0.13$ & $26.80 \pm 0.10$ \\
\hline $1440-0303$ & 18.63 & 0.754 & $-24.42 \pm 0.48$ & $<0.45$ & $<0.56$ & $<24.03$ \\
\hline $1441+0134$ & 18.45 & 0.753 & $-24.60 \pm 0.47$ & $<0.40$ & 0.44 & $<23.99$ \\
\hline $1443-0207$ & 18.49 & 0.397 & $-23.20 \pm 0.41$ & $0.58 \pm 0.16$ & $0.60 \pm 0.21$ & $23.59 \pm 0.14$ \\
\hline $1444-0019$ & 18.20 & 0.697 & $-24.67 \pm 0.46$ & $<0.49$ & $<0.43$ & $<24.00$ \\
\hline
\end{tabular}


TABLE 1-Continued

\begin{tabular}{|c|c|c|c|c|c|c|}
\hline $\begin{array}{l}\text { Source } \\
(1)\end{array}$ & $\begin{array}{c}B \\
(2)\end{array}$ & $\begin{array}{c}z \\
(3)\end{array}$ & $\begin{array}{l}M_{B} \\
(4)\end{array}$ & $\begin{array}{c}S_{8.4} \\
(\mathrm{mJy}) \\
(5)\end{array}$ & $\begin{array}{l}\log R_{8.4} \\
\quad(6)\end{array}$ & $\begin{array}{c}\log L_{8.4} \\
\left(\mathrm{WHz}^{-1}\right) \\
(7)\end{array}$ \\
\hline $1444-0300$ & 18.46 & 0.549 & $-23.90 \pm 0.43$ & $<0.43$ & 0.48 & $<23.74$ \\
\hline $1445+0222$ & 18.54 & 0.775 & $-24.57 \pm 0.48$ & $<0.35$ & 0.41 & $<23.95$ \\
\hline $1446+0027$ & 18.61 & 0.832 & $-24.66 \pm 0.49$ & $<0.74$ & 0.76 & $<24.33$ \\
\hline $1446+0218$ & 17.89 & 0.672 & $-24.90 \pm 0.44$ & $<0.37$ & 0.19 & $<23.85$ \\
\hline $2158-1657$ & 18.47 & 0.475 & $-23.60 \pm 0.43$ & $<0.42$ & 0.46 & $<23.61$ \\
\hline $2159-1906$ & 18.22 & 0.364 & $-23.30 \pm 0.42$ & $<0.47$ & 0.40 & $<23.43$ \\
\hline $2207-1703$ & 18.43 & 0.371 & $-23.13 \pm 0.42$ & $<0.42$ & 0.43 & $<23.39$ \\
\hline $2212-1634$ & 17.80 & 0.296 & $-23.31 \pm 0.41$ & $<0.42$ & 0.17 & $<23.20$ \\
\hline $2214-1903$ & 18.18 & 0.396 & $-23.51 \pm 0.42$ & $<0.45$ & 0.37 & $<23.48$ \\
\hline $2235+0054$ & 18.57 & 0.529 & $-23.71 \pm 0.42$ & $2.67 \pm 0.43$ & $1.31 \pm 0.20$ & $24.50 \pm 0.10$ \\
\hline $2235-0112$ & 18.21 & 0.361 & $-23.29 \pm 0.39$ & $<0.45$ & $<0.37$ & $<23.40$ \\
\hline $2348+0210$ & $17.67^{\mathrm{m}}$ & 0.504 & $-24.73 \pm 0.18$ & $33.77 \pm 0.45$ & $1.96 \pm 0.10$ & $25.56 \pm 0.07$ \\
\hline $2350-0045 B$ & 18.43 & 0.764 & $-24.65 \pm 0.47$ & $<0.43$ & $<0.46$ & $<24.02$ \\
\hline $2351-0036$ & $18.71^{\mathrm{m}}$ & 0.460 & $-23.61 \pm 0.22$ & $279.0 \pm 3.4$ & $3.25 \pm 0.11$ & $26.40 \pm 0.07$ \\
\hline $2352+0025$ & 17.81 & 0.271 & $-23.12 \pm 0.38$ & $<0.43$ & $<0.17$ & $<23.13$ \\
\hline $2353+0032$ & 18.59 & 0.558 & $-23.80 \pm 0.43$ & $<0.46$ & 0.56 & $<23.79$ \\
\hline $2357-0014$ & 17.86 & 0.479 & $-24.22 \pm 0.40$ & $<0.41$ & 0.21 & $<23.60$ \\
\hline
\end{tabular}

${ }^{\text {II }}$ Previously reported in Paper II. The derived quantities $M_{B}$ and $\log R_{8.4}$, including errors, have been updated using the numbers in Table 2. Redshift and $8.4 \mathrm{GHz}$ flux and luminosity, and in most cases apparent $B$ magnitude, are retained from Paper II. A new $B$ magnitude, if available, is listed in Column 2 and flagged (see below).

${ }^{\mathrm{m}}$ The apparent $B$ magnitude was determined from the new photometry in Table 2. Entries without this flag have $B$ values calculated from original $B_{J}$ magnitudes, as described in Paper II. 

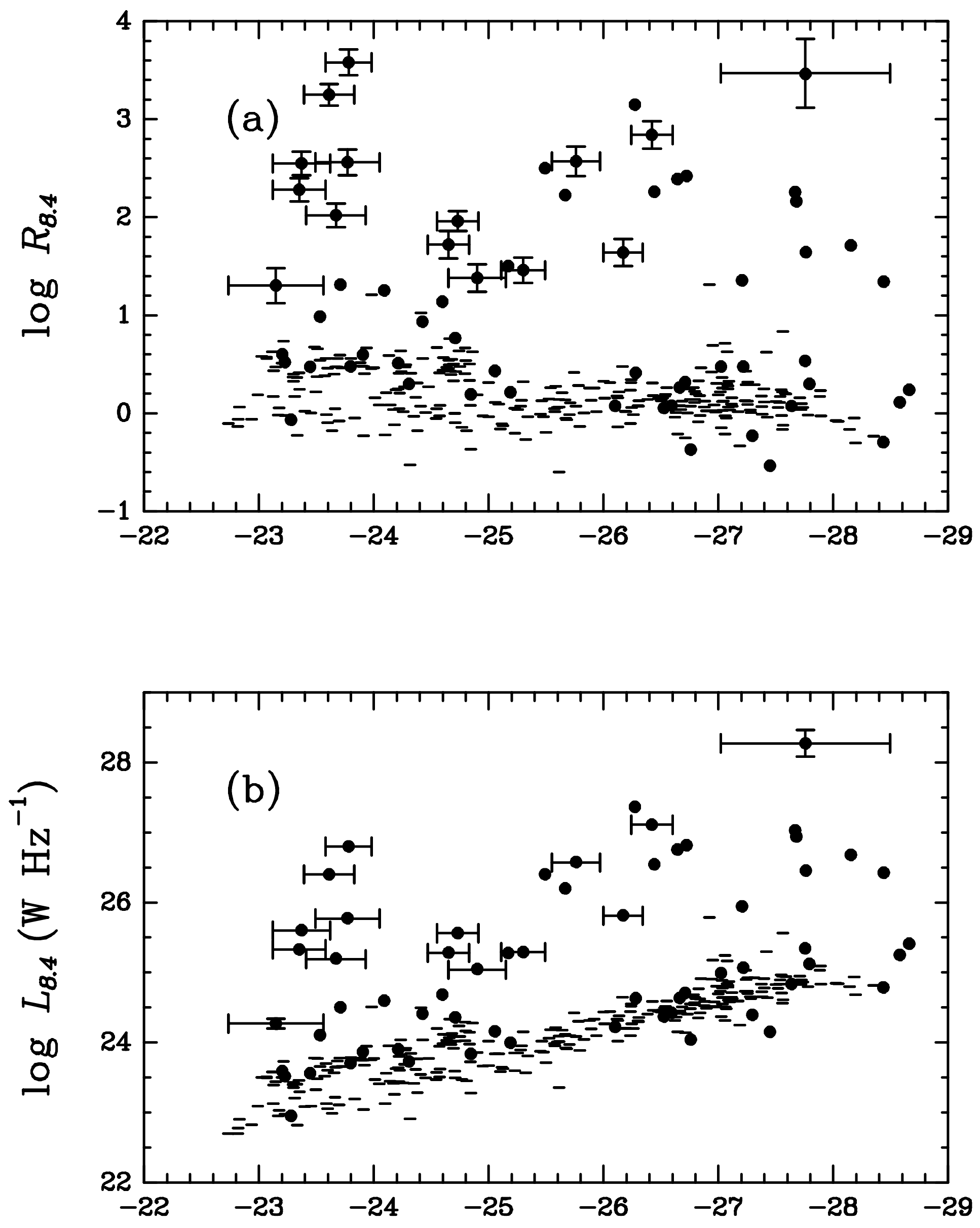
TABLE 2

Optical Data for Selected Radio-Loud LBQS Quasars

\begin{tabular}{lcccccc}
\hline \hline $\begin{array}{c}\text { Name } \\
(1)\end{array}$ & $\begin{array}{c}V \\
(2)\end{array}$ & $\begin{array}{c}B-V \\
(3)\end{array}$ & $\begin{array}{c}V-R \\
(4)\end{array}$ & \multicolumn{1}{c}{$\begin{array}{c}\alpha \\
(5)\end{array}$} & $\begin{array}{c}\text { MgII } \\
(7)\end{array}$ & $\begin{array}{c}\text { CIII }] \\
(8)\end{array}$ \\
\hline $1137+0110^{\mathrm{b}}$ & $17.71 \pm 0.09$ & $\ldots$ & $0.30 \pm 0.17$ & $-0.25 \pm 0.13$ & $97 \pm 10$ & $61 \pm 4$ \\
$1138+0003$ & $18.30 \pm 0.02$ & $0.05 \pm 0.02$ & $0.16 \pm 0.02$ & $1.09 \pm 0.19$ & $79 \pm 3$ & $\ldots$ \\
$1148-0033^{\mathrm{b}}$ & $17.96 \pm 0.11$ & $\ldots$ & $0.37 \pm 0.20$ & $-0.37 \pm 0.11$ & $53 \pm 4$ & $\ldots$ \\
$1211+0848^{\mathrm{b}}$ & $17.46 \pm 0.06$ & $\ldots$ & $0.20 \pm 0.10$ & $-0.56 \pm 0.10$ & $59 \pm 5$ & $\ldots$ \\
$1218+1734$ & $18.30 \pm 0.03$ & $0.06 \pm 0.04$ & $0.36 \pm 0.04$ & $0.35 \pm 0.14$ & $14 \pm 1$ & $\ldots$ \\
$1222+1235$ & $17.86 \pm 0.04$ & $0.07 \pm 0.04$ & $0.34 \pm 0.04$ & $1.00 \pm 0.18$ & $78 \pm 3$ & $\ldots$ \\
$1229-0207^{\mathrm{b}}$ & $16.76 \pm 0.07$ & $\ldots$ & $0.18 \pm 0.13$ & $-0.23 \pm 0.12$ & $95 \pm 8$ & $31 \pm 4$ \\
$1230-0015^{\mathrm{b}}$ & $17.63 \pm 0.12$ & $\ldots$ & $0.00 \pm 0.22$ & $0.66 \pm 0.16$ & $15 \pm 1$ & $\ldots$ \\
$1308-0111$ & $18.34 \pm 0.02$ & $\ldots$ & $0.13 \pm 0.02$ & $-0.04 \pm 0.14$ & $71 \pm 6$ & $46 \pm 6$ \\
$1430-0046^{\mathrm{a}, \mathrm{b}}$ & $17.31 \pm 0.05$ & $\ldots$ & $0.45 \pm 0.09$ & $-0.47 \pm 0.15$ & $59 \pm 10$ & $35 \pm 5$ \\
$1438+0210$ & $19.01 \pm 0.03$ & $\ldots$ & $0.25 \pm 0.03$ & $-0.68 \pm 0.11$ & $30 \pm 6$ & $\ldots$ \\
$2348+0210^{\mathrm{a}}$ & $17.33 \pm 0.04$ & $0.34 \pm 0.04$ & $\ldots$ & $-0.29 \pm 0.18$ & $36 \pm 4$ & $\ldots$ \\
$2351-0036^{\mathrm{a}}$ & $18.30 \pm 0.05$ & $0.41 \pm 0.06$ & $\cdots$ & $-0.50 \pm 0.24$ & $41 \pm 7$ & $\ldots$ \\
& & & & & & \\
\hline
\end{tabular}

${ }^{a}$ No recent long slit CCD spectra are available for these quasars. Archival spectra were used to calculate spectral indices, $k$-corrections, and equivalent widths.

${ }^{\mathrm{b}} V$ and $V-R$ are from a large multiband study of the LBQS currently in progress. 

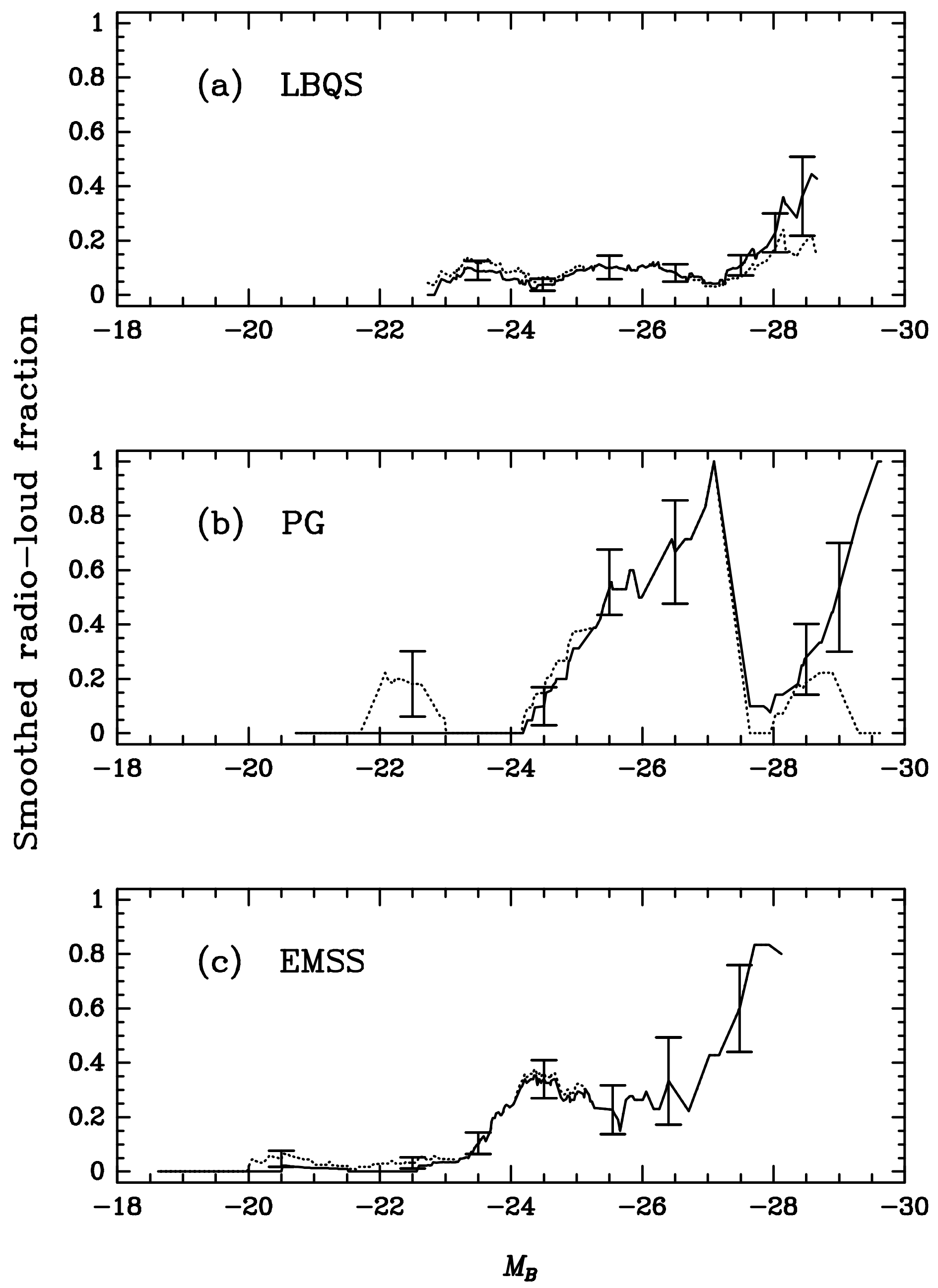

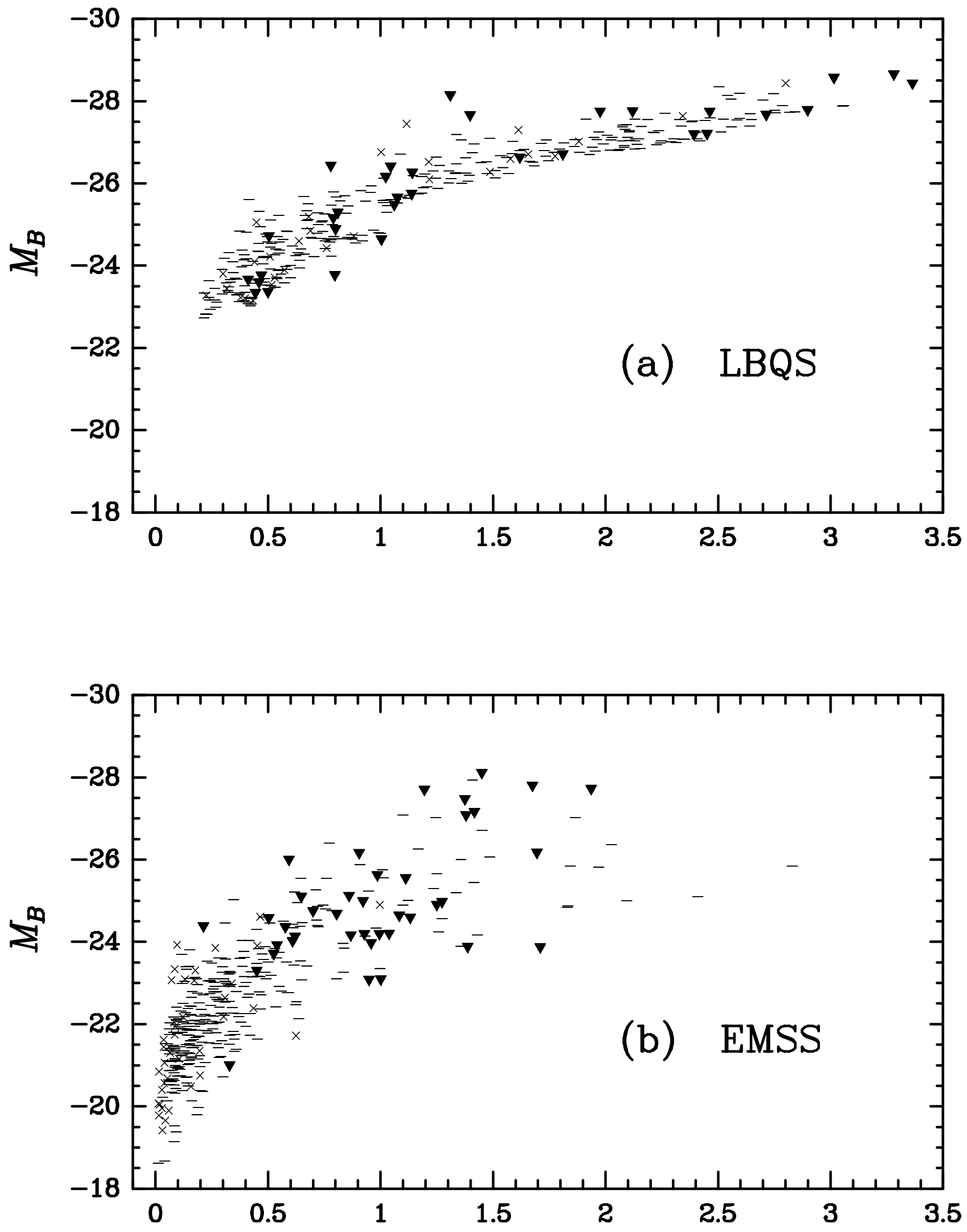

Redshift 


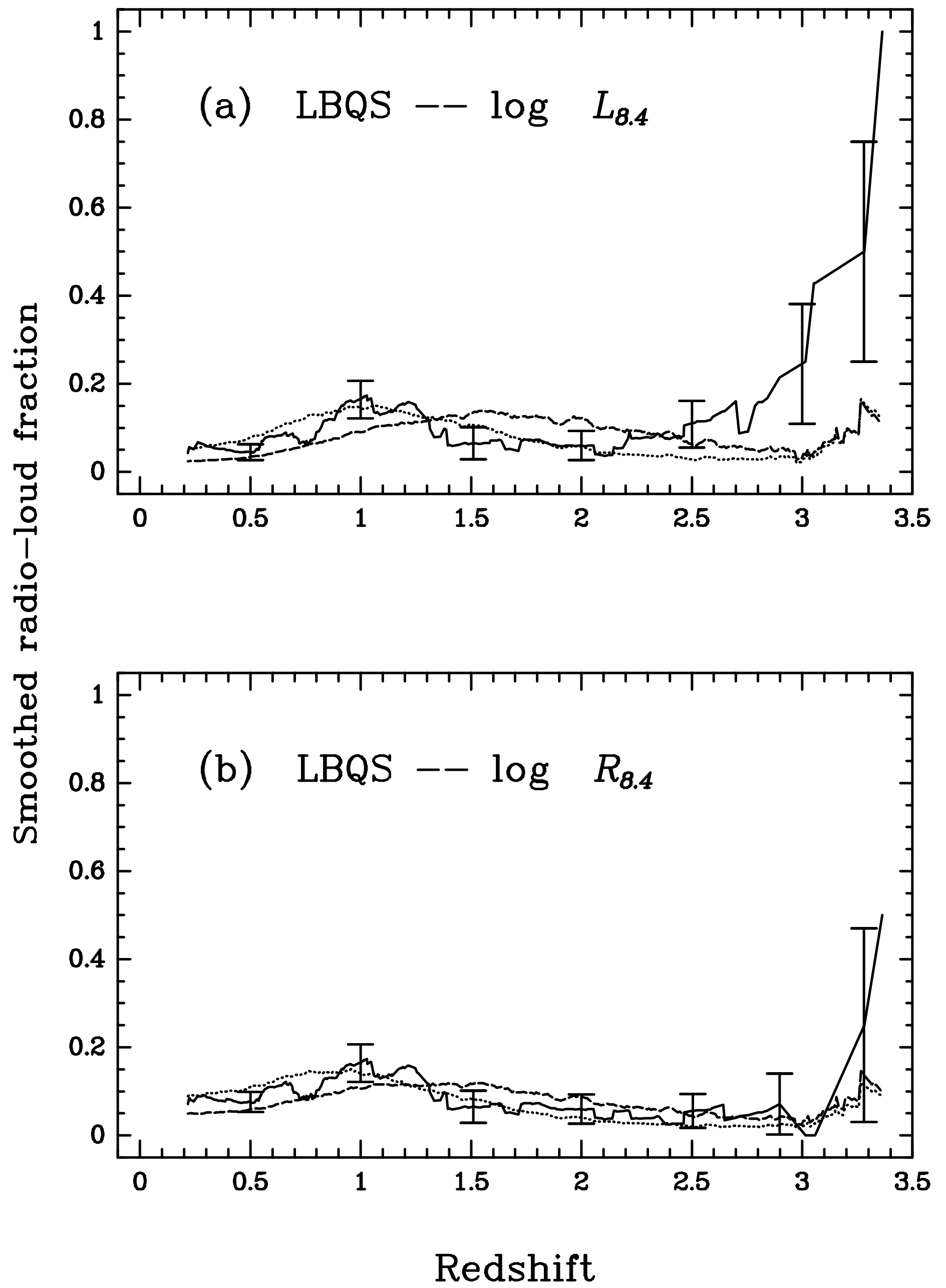




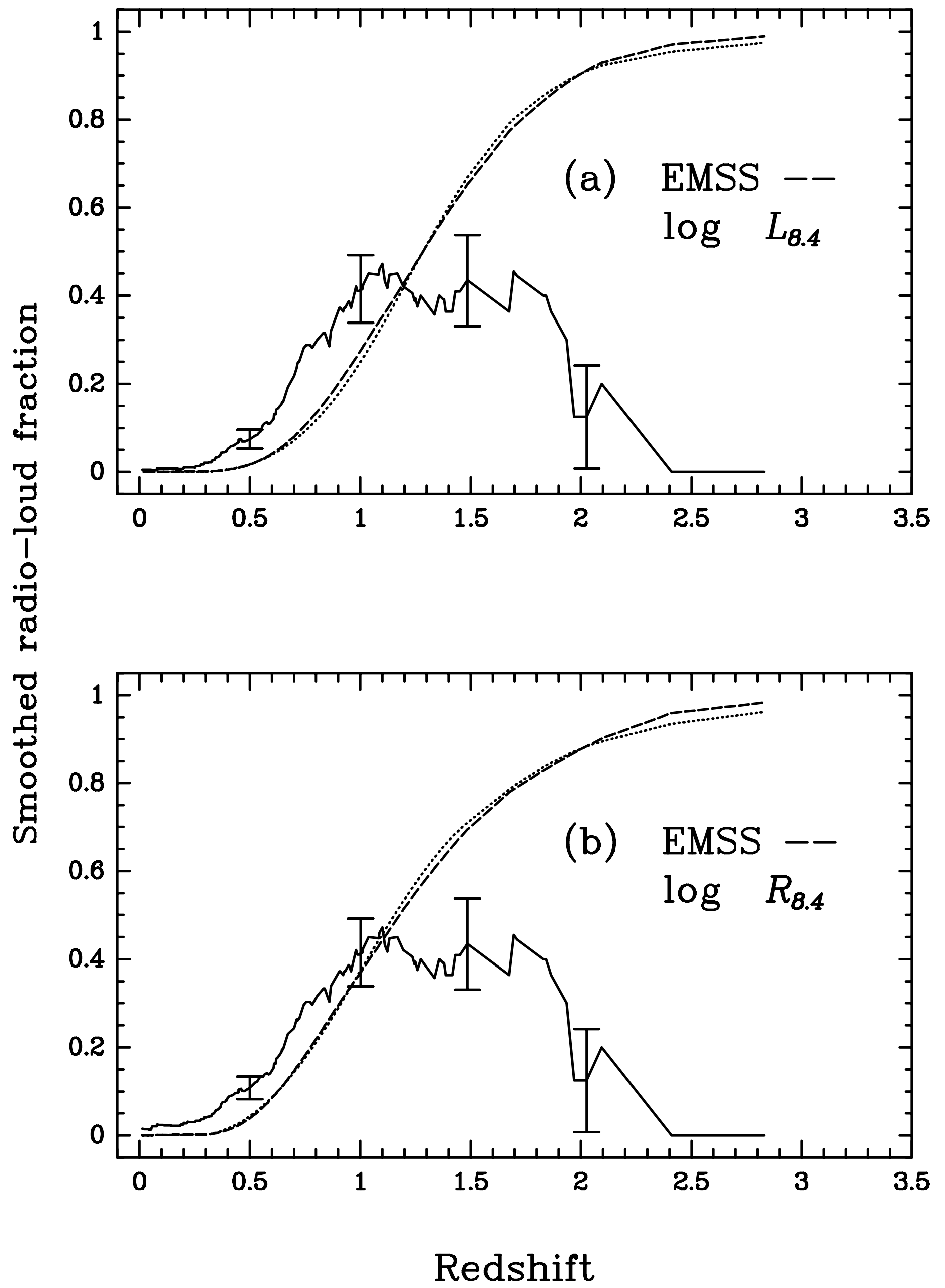



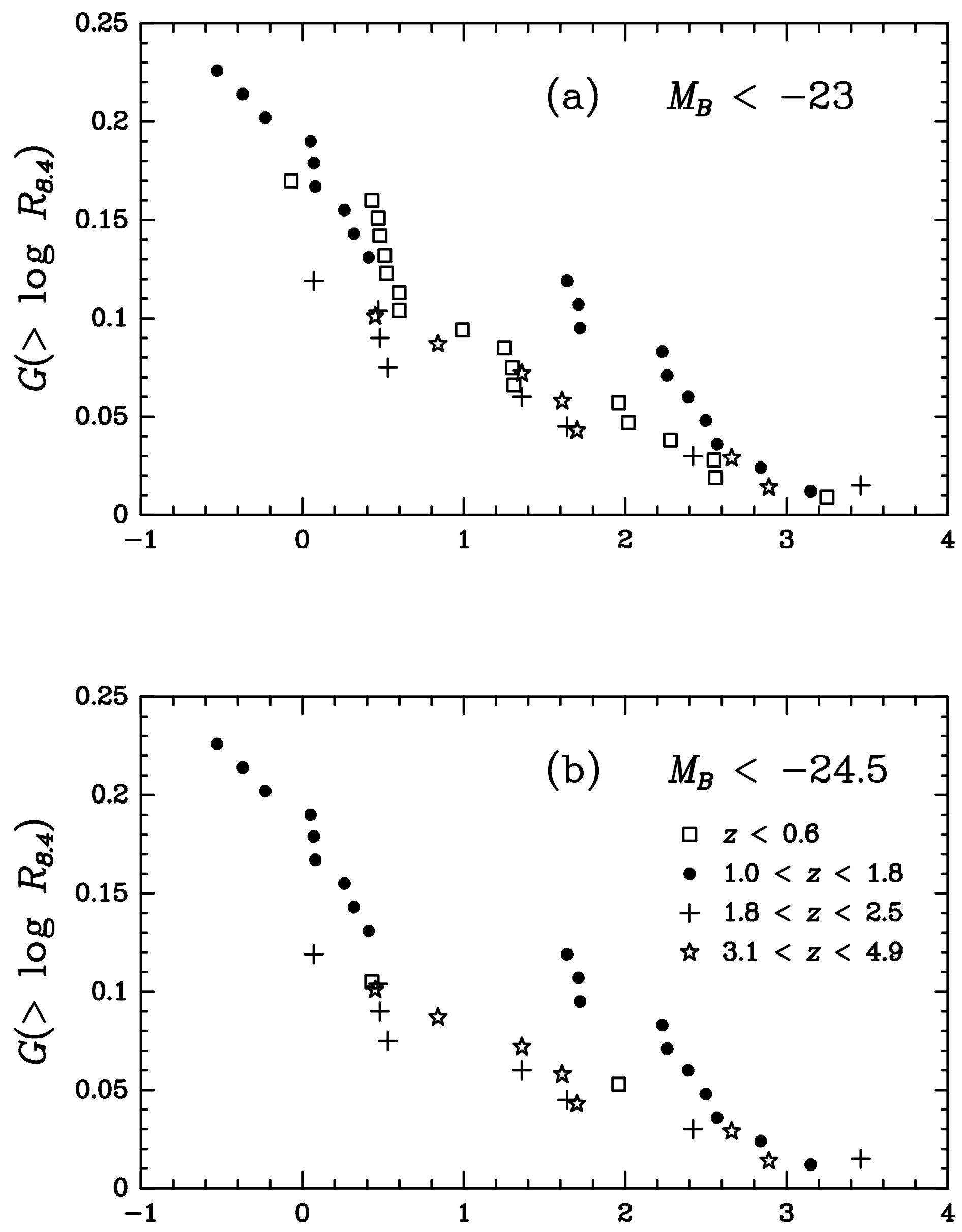

$\log R_{8.4}$ 